NASA Contractor Report 191528

ICASE Report No. 93-61

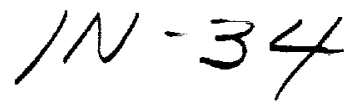

190190

$48 P$

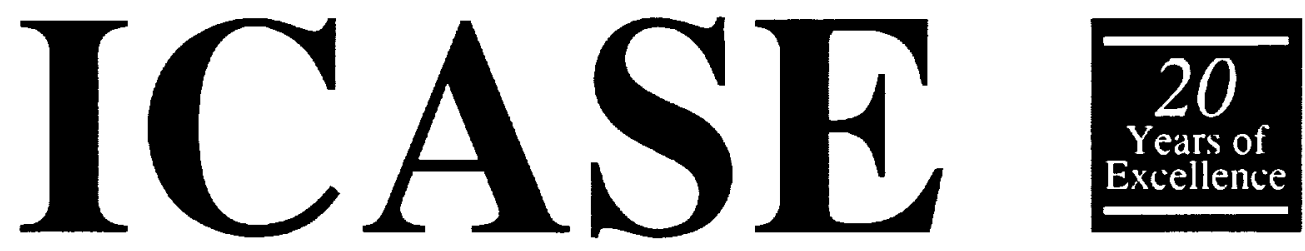

ON THE INTERACTION BETWEEN THE SHOCK WAVE ATTACHED TO A WEDGE AND FREESTREAM DISTURBANCES

Peter W. Duck

D. Glenn Lasseigne

M. Y. Hussaini

NASA Contract Nos. NAS 1-19480 and NAS 1-18605

August 1993

Institute for Computer Applications in Science and Engineering NASA Langley Research Center

Hampton, Virginia 23681-0001

Operated by the Universities Space Research Association

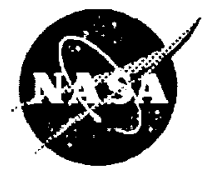

$\begin{array}{lll}\infty & & \\ 1 & & 0 \\ 1 & n & 0 \\ 1 & 0 & 0 \\ 2 & 4 & 0 \\ 2 & 5 & 0\end{array}$

National Aeronautics and Space Administration Langley Research Center Hampton, Virginia 23681-0001

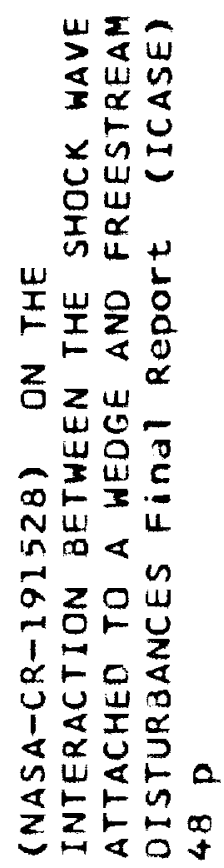




\section{ICASE Fluid Mechanics}

Due to increasing research being conducted at ICASE in the field of fluid mechanics, future ICASE reports in this area of research will be printed with a green cover. Applied and numerical mathematics reports will have the familiar blue cover, while computer science reports will have yellow covers. In all other aspects the reports will remain the same; in particular, they will continue to be submitted to the appropriate journals or conferences for formal publication. 


\title{
ON THE INTERACTION BETWEEN THE SHOCK WAVE ATTACHED TO A WEDGE AND FREESTREAM DISTURBANCES
}

\author{
Peter W. Duck \\ Department of Mathematics \\ University of Manchester \\ D. Glenn Lasseigne ${ }^{1}$ \\ Department of Mathematics and Statistics \\ Old Dominion University \\ Norfolk, Virginia 23529 \\ M. Y. Hussaini ${ }^{1}$ \\ Institute for Computer Applications in Science and Engineering \\ NASA Langley Research Center \\ Hampton, Virginia 23665
}

\begin{abstract}
We present a study of the interaction of small amplitude, unsteady, freestream disturbances with a shock wave induced by a wedge in supersonic flow. These disturbances may be acoustic waves, vorticity waves, or entropy waves (or indeed a combination of all three). Their interactions then generate behind the shock disturbances of all three classes, an aspect that is investigated in some detail, our motivation here being to investigate possible mechanisms for boundary-layer receptivity, caused through the amplification and modification of freestream turbulence through the shock-body coupling. Also, the possibility of enhanced mixing owing to additional vorticity produced by the shock-body coupling is investigated.
\end{abstract}

\footnotetext{
${ }^{1}$ This research was supported by the National Aeronautics and Space Administration under NASA Contract Nos. NAS1-19480 and NAS1-18605 while the authors were in residence at the Institute for Computer Applications in Science and Engineering (ICASE), NASA Langley Research Center, Hampton, VA 236810001 .
} 


\section{Introduction.}

Boundary-layer receptivity (i.e the growth of instabilities within the boundary layer, triggered by some form of external disturbances) has been observed in supersonic wind tun-

nel experiments in the presence of sound waves radiated by turbulent tunnel-wall boundary layers or by freestream turbulence. In such situations, the acoustic wave mainstream disturbances are first processed by the shock wave induced by the body in supersonic stream, and then interact with the boundary layer to cause instability (if at all). In this paper, we study the former phase of how a shock in the presence of the body processes mainstream disturbances. The latter phase of these disturbances internalizing in the boundary layer will be subject of future study. The shock-body combination is shown to produce a wide spectrum of wavelengths from monochromatic waves, i.e a freestream disturbance with a single wavelength; in particular, we note the production of shorter wavelength disturbances than would be expected from the free shock-disturbance interaction. Then these shorter wavelength perturbations resulting from the shock-body combination would go on to interact with the boundary layer on the surface of the body, leading to possible instability. In the case of turbulence-shock-body interaction, it is noted that the turbulence itself is composed of a continuous spectrum of wavelengths and the interaction would produce even more short-wavelength disturbances than the single wavelength disturbance considered here. Such interactions could possibly enhance mixing of a multi-component flow owing to the amplification and generation of vorticity behind the shock. Again, it is found that the shock-body combination efficiently processes the freestream disturbance and generates a vorticity profile behind the shock which are larger than those arising from the interaction of the free shock with mainstream disturbances.

To investigate these phenomena, we consider the model problem of a wedge in a uniform supersonic stream. In the presence of the wedge, the supersonic flow abruptly changes direction through an oblique shock wave, details of which are determined by the Rankine-Hugoniot conditions. This paper is concerned with the response of the overall flow field to general, small amplitude disturbances in the freestream that are convected 
through the shock wave. In order to quantify this response, we consider isolated single wavelength disturbances of acoustic type, vorticity type or entropy type. In particular, we are concerned with the production of a continuous spectrum of wavelengths produced in the flow behind the shock in the presence of the wedge. Moore (1954), Ribner (1953) and McKenzie and Westphal (1968) have investigated the processing of single wavelength disturbances by freely propagating shocks, and note that through the interaction of the shock and the freestream disturbance, single wavelength entropy, acoustic and vorticity disturbances are all generally produced behind the shock in response to an isolated acoustic disturbance, or to an isolated entropy disturbance or to an isolated vorticity disturbance. With the presence of the wedge, the flow behind the shock is no longer comprised of single wavelength acoustic, entropy and vorticity disturbances. In order to satisfy the condition of zero normal velocity at the wedge surface as well as the condition that the shock remain attached to the apex of the wedge, a continuous spectrum of wavelengths for the acoustic, entropy and vorticity disturbances exists behind the shock. It is this more complicated disturbance pattern that goes on to interact with the boundary layer developing along the surface of the wedge, possibly initiating new receptivity mechanisms.

Limited work appears to have been done on the theoretical/computational approach to the interaction between shock waves and boundary-layer instabilities, although most of this work has focused on the wedge problem. A fairly restrictive (and somewhat ad hoc) model was presented by Petrov (1984), using inviscid linear stability equations, and somewhat heuristic conditions on the shock (which was also assumed to lie at the edge of the boundary layer). Cowley and Hall (1988) presented an asymptotic model, applicable to three-dimensional viscous modes of instability, with appropriately simplified conditions applied on the shock surface (derived from the Rankine-Hugoniot conditions), which was taken to lie just outside of the boundary layer. Chang, Malik and Hussaini (1990) considered the viscous small disturbance equations with parallel mean flow assumption, using the full inviscid Rankine-Hugoniot conditions on the shock.

Here we take the equations governing the gas flow both ahead of and behind the shock to be the Euler equations (neglecting the boundary layer on the wedge in the first order of approximation) along with the ideal gas law. The no mass flux condition is imposed on the 
wedge surface, so that the normal component of the flow must have the same velocity as the velocity of the wedge normal to its surface. Furthermore, it is required that the shock remains attached to the apex of the wedge. The analysis is accomplished by linearizing the Euler equations about the base state and applying the Rankine-Hugoniot conditions at the mean position of the shock wave.

Carrier (1949a) and Van Dyke (1953) first investigated the response of the inviscid flow field to time-periodic oscillations of the wedge. Their interest was in the surface pressure distribution and the resultant forces and moments, and their relevance to oscillating airfoils. The related problem of freestream turbulence amplification caused through interactions with shock waves has been considered by Anyiwo and Bushnell (1982), Hussaini, Collier and Bushnell(1986), Meadows, Kumar,and Hussaini (1991), and Kumar, Bushnell and Hussaini (1989) who confirmed the important result found in Moore (1951), Ribner (1954) and McKenzie and Westphal (1968) that a pure acoustic, vorticity, or entropy wave, upon interacting with a plane shock wave, generally generates all three classes of disturbance downstream. The interaction of a shear wave with a detonation wave induced by a wedge in a supersonic flow, as well as the response of the oblique detonation to oscillations of the wedge was considered by Lasseigne and Hussaini (1992). In studying the interaction of a shear wave with the detonation, they assumed that a weak steady sinusoidal vorticity wave is obliquely convected through an overdriven detonation attached to a wedge. Their concern was with the response, measured by the deviation of the detonation position from its unperturbed state and by the vorticity and pressure at the detonation, as the degree of overdrive is increased. They compared the flow in the presence of the wedge with the unobstructed flow field considered in Jackson, Kapila, \& Hussaini (1990). In particular, for this special type of disturbance (i.e. steady), they found an infinite discrete spectrum of disturbances to exist behind the shock.

The present study also raises other important, broader issues, notably the stability of the shock on the wedge. It is generally accepted that the so-called "strong shock" solution (Liepmann and Roshko 1957) is unstable (see Levinson 1945, Carrier 1949b, Henderson and Atkinson 1976, Rusanov and Sharakshanae 1980, and Salas and Morgan 1982), whilst the proof for the stability of the "weak shock" solution has largely been either numerical 
(Rusanov and Sharakshanae 1980, and Salas and Morgan 1982) or subject to some restrictions. The work of Henderson and Atkinson (1976) considered just finite length wedges to "avoid unbounded velocity downstream" (sic) whilst Carrier (1949b) did "not worry about convergence in the large" (sic) when considering series solutions. One of the aims of this paper is to place the evidence for the stability of flows in which the flow downstream of the shock is supersonic on a much firmer footing. Here we shall remove Henderson and Atkinsons's (1970) restriction and pay particular attention to the convergence of series solutions.

The layout of the paper is as follows. In Section 2 we consider the three distinct classes of disturbances upstream of the shock, and then in Section 3, the analytic solution for the downstream development of these disturbances in the region bounded by the shock and the wedge surface is obtained. In Section 4, we examine various aspects of the analytical solution. These raise important questions regarding the stability of the shock itself. We show that the weak shock solution is stable to these imposed disturbances, provided that the downstream flow is supersonic in nature, in line with previous (mostly numerical and experimental) studies. We also examine the behaviour of the far-downstream flow which suggests an important physical decomposition of the solution. The physical aspects of the decomposition is examined in Section 5 and the responses to the various types of imposed disturbances are quantified. Our conclusions are given in Section 6.

\section{Formulation}

Throughout we will denote quantities upstream of the shock by subscript 1 and downstream by subscript 2 . We take the wedge surface to make an angle $\theta$ with respect to the on-coming flow, with $\left(x^{*}, y^{*}\right)$ the coordinates parallel and perpendicular to the upstream base flow (respectively) which has magnitude $U_{1}^{*}$, Mach Number $M_{1}$, density $\rho_{1}^{*}$, and temperature $T_{1}^{*}$. We assume that the ratio of specific heats $\gamma$ is constant. The velocity vector is written as $U_{1}^{*}(u, v)$ with respect to $\left(x^{*}, y^{*}\right)$ coordinates. The density field is written as $\rho_{1}^{*} \rho$, pressure as $\rho_{1}^{*} R^{*} T_{1}^{*} p$, where $R^{*}$ denotes the gas constant and the temperature field as $T_{1}^{*} T$. 
The Rankine-Hugoniot relations provides a link between conditions upstream and conditions downstream of the shock, and in particular leads to the following classical result in gas dynamics (e.g Liepmann and Roshko 1957),

$$
\frac{\tan (\beta-\theta)}{\tan \beta}=\frac{\bar{U}_{2}}{\bar{u}_{1}}=\frac{\rho_{1}}{\rho_{2}}=\frac{(\gamma-1) M_{1}^{2} \sin ^{2} \beta+2}{(\gamma+1) M_{1}^{2} \sin ^{2} \beta},
$$

where $\beta$ is the angle between the shock and the wedge centerline, and $\bar{u}_{1}$ and $\bar{u}_{2}$ are the non-dimensional velocity components perpendicular to the shock.

It is well known (Liepmann and Roshko 1957) that for a given upstream Mach number $M_{1}$ and $\theta<\theta_{\max }\left(M_{1}\right)$ equation (2.1) admits two possible solutions for $\beta$ : the larger value corresponding to the so called "strong shock" solution, which is characterized by subsonic flow behind the shock in all cases and the smaller value corresponding to the so-called weak solution which is characterized by the flow being generally supersonic behind the shock, except for a small region of subsonic flow for $\theta$ close to $\theta=\theta_{\max }$. For $\theta>\theta_{\max }$, no attached-shock solutions to the problem exist. Attached shock solutions all have the property of uniform downstream flow directed parallel to the wedge surface.

Small amplitude disturbances upstream of the shock may be classified into three distinct classes (see McKenzie and Westphal 1968, for example). Taking $\epsilon$ to be the measure of the amplitude, and hence a small parameter, we have:

(i) Acoustic waves: These are characterized by having a pressure perturbation and the resultant perturbations in velocity, density and temperature; however, the waves carry no change in entropy and have no vorticity. In this case the disturbance field upstream of the shock is given by

$$
\begin{gathered}
p=1+\epsilon \hat{E}+O\left(\epsilon^{2}\right), \\
u=1-\frac{\alpha_{1} \epsilon \hat{E}}{\gamma M_{1}^{2}\left(\alpha_{1}+\omega\right)}+O\left(\epsilon^{2}\right), \\
v=-\frac{\alpha_{2} \epsilon \hat{E}}{\gamma M_{1}^{2}\left(\alpha_{1}+\omega\right)}+O\left(\epsilon^{2}\right), \\
\rho=1+\frac{\epsilon \hat{E}}{\gamma}+O\left(\epsilon^{2}\right),
\end{gathered}
$$




$$
T=1-\frac{\epsilon(\gamma-1) \hat{E}}{\gamma}+O\left(\epsilon^{2}\right)
$$

where $\hat{E}$ is the normal mode exponential

$$
\hat{E}=\exp \left\{i \alpha_{1} x_{1}+i \alpha_{2} y_{1}+i \omega t\right\},
$$

and

$$
\omega=-\alpha_{1} \pm \frac{1}{M_{1}}\left(\alpha_{1}^{2}+\alpha_{2}^{2}\right)^{1 / 2}
$$

is the frequency of the disturbance for the given wavenumbers. The coordinates $x_{1}$ and $y_{1}$ are parallel and perpendicular to the upstream flow respectively, suitably nondimensionalized (this can be accomplished by using one of the wavelengths of the disturbance as a characteristic length scale, e.g. setting $\alpha_{1}$, say, to unity). The choice of frequency $\omega$ and also the nature of the solution (2.2)-(2.6) arises from solving the irrotational flow problem upstream of the shock. We shall refer to modes corresponding to the negative sign in (2.8) as so-called "fast modes", and to the positive sign in (2.8) as the so-called "slow modes".

(ii) Vorticity waves: These are characterized by having no density, temperature or pressure components to the disturbance to $O(\epsilon)$. As such, the upstream field can be written

$$
\begin{gathered}
u=1+\epsilon \hat{E}+O\left(\epsilon^{2}\right), \\
v=-\epsilon \frac{\alpha_{1}}{\alpha_{2}} \hat{E}+O\left(\epsilon^{2}\right), \\
\rho, T, p=1+O\left(\epsilon^{2}\right),
\end{gathered}
$$

together with

$$
\omega=-\alpha_{1},
$$

with $\hat{E}$ having the same definition as in (2.7).

(iii) Entropy waves: These are characterized by having no pressure or velocity components to the disturbance to $O(\epsilon)$. As such, the upstream field can be written

$$
\rho=1+\epsilon \hat{E}+O\left(\epsilon^{2}\right)
$$




$$
\begin{gathered}
T=1-\epsilon \hat{E}+O\left(\epsilon^{2}\right), \\
u, p=1+O\left(\epsilon^{2}\right), \\
v=O\left(\epsilon^{2}\right),
\end{gathered}
$$

together with $\omega$ defined as in (2.12) and $\hat{E}$ defined as in (2.7).

In the following section we go on to consider the interaction between these waves and the shock attached to the wedge. Fortunately, in spite of their distinct features, the analysis is fundamentally the same in all three cases, and we shall see how just one of these waves upstream of the shock generally produces a combination of all three modes of disturbance downstream of the shock; however, unlike the situation where no wedge is present, the downstream disturbance is not restricted to a single wavelength.

\section{The Solution Downstream of the Shock}

Downstream of the shock, the flow variables may be written

$$
\begin{gathered}
\rho=\rho_{2}+\epsilon \tilde{\rho}+O\left(\epsilon^{2}\right), \\
p=p_{2}+\epsilon \tilde{p}+O\left(\epsilon^{2}\right), \\
T=T_{2}+\epsilon \tilde{T}+O\left(\epsilon^{2}\right) .
\end{gathered}
$$

The non-dimensional coordinates parallel and perpendicular to the wedge are taken as $x_{2}$ and $y_{2}$ respectively whilst the velocity components in the $x_{2}$ and $y_{2}$ direction are written as $U_{2}+\epsilon \tilde{u}+O\left(\epsilon^{2}\right)$ and $\epsilon \tilde{v}+O\left(\epsilon^{2}\right)$ respectively. The governing equations at $O(\epsilon)$ may be written

$$
\begin{gathered}
\tilde{\rho}_{t}+U_{2} \tilde{\rho}_{x_{2}}+\rho_{2} \tilde{u}_{x_{2}}+\rho_{2} \tilde{v}_{y_{2}}=0, \\
\rho_{2}\left\{\tilde{u}_{t}+U_{2} \tilde{u}_{x_{2}}\right\}+\frac{1}{\gamma M_{1}^{2}} \tilde{p}_{x_{2}}=0, \\
\rho_{2}\left\{\tilde{v}_{t}+U_{2} \tilde{v}_{x_{2}}\right\}+\frac{1}{\gamma M_{1}^{2}} \tilde{y}_{y_{2}}=0, \\
\rho_{2}\left\{\tilde{T}_{t}+U_{2} \tilde{T}_{x_{2}}\right\}-\frac{\gamma-1}{\gamma}\left\{\tilde{p}_{t}+U_{2} \tilde{p}_{x_{2}}\right\}=0
\end{gathered}
$$




$$
\tilde{p}=\rho_{2} \tilde{T}+T_{2} \tilde{\rho}
$$

The general solution procedure is based on that followed by Carrier (1949a) and Van Dyke (1953). It is found convenient to first split the solution into two components, corresponding to acoustic waves and vorticity waves. Specifically, we write

$$
\begin{aligned}
& \tilde{u}=\phi_{x_{2}}+E_{y_{2}}, \\
& \tilde{v}=\phi_{y_{2}}-E_{x_{2}},
\end{aligned}
$$

where $\phi=\phi\left(x_{2}, y_{2}, t\right)$ represents the acoustic mode and $E=E\left(x_{2}, y_{2}, t\right)$ represents the vorticity mode.

Substituting these forms into (3.5)-(3.6), differentiating and combining appropriately yields the following equations

$$
\begin{gathered}
\nabla^{2}\left\{\phi_{t}+U_{2} \phi_{x_{2}}+\frac{\tilde{p}}{\gamma M_{1}^{2} \rho_{2}}\right\}=0 \\
\nabla^{2}\left\{E_{t}+U_{2} E_{x_{2}}\right\}=0
\end{gathered}
$$

Solving (3.11) and (3.12) implies the introduction of two arbitrary harmonic functions (see the footnote in Hui 1969). Equations (3.11) and (3.12) taken together represent a sixth order system which is derived from the second order system (3.5)-(3.6), and therefore without loss of generality, we set these two arbitrary harmonic functions to zero and note that all physical conditions at the shock and the wedge boundary are satisfied by the resulting solution. Using (3.4), (3.7) and (3.8), it is then possible to show that $\phi$ satisfies the following equation

$$
\nabla^{2} \phi=\frac{1}{a_{s}^{2}}\left\{\phi_{t \imath}+2 U_{2} \phi_{x_{2} t}+U_{2}^{2} \phi_{x_{2} x_{2}}\right\}
$$

with

$$
\tilde{p}=-\frac{\gamma p_{2}}{a_{s}^{2}}\left(\phi_{t}+U_{2} \phi_{x}\right)
$$

where

$$
a_{s}=\frac{T_{2}^{1 / 2}}{M_{1}}
$$


is the nondimensional sound speed behind the shock. A further quantity is also required to describe the problem fully, namely $\psi^{\prime}\left(y_{2}, t\right)$, the displacement of the shock wave.

It is possible to write the general solution for $\phi$ and $E$ which is bounded at the tip of the wedge as

$$
\begin{aligned}
& \phi=e^{i \omega t-\frac{i \omega U_{2} x_{2}}{U_{2}^{2}-a_{j}^{2}}} \sum_{\nu=0}^{\infty}\left\{a_{\nu} \cosh \left(\nu \theta_{2}\right)+b_{\nu} \sinh \left(\nu \theta_{2}\right)\right\} J_{\nu}(\hat{k} r) \\
& E=e^{i \omega t-\frac{i \omega x_{2}}{U_{2}}-\frac{i \omega y_{2} \lambda a_{a}^{2}}{U_{2}\left(U_{2}^{2}-a_{0}^{2}\right)}} \sum_{\nu=0}^{\infty} c_{\nu} J_{\nu}\left(\hat{k} \hat{\xi} y_{2}\right) \\
& \psi^{\prime}=e^{i \omega t-\frac{i \omega_{2} \lambda_{y_{2}}}{U_{2}^{2}-a_{2}^{2}}} \sum_{\nu=0}^{\infty} d_{\nu} J_{\nu}\left(\hat{k} \hat{\xi} y_{2}\right)
\end{aligned}
$$

where

$$
\begin{gathered}
\hat{k}=\frac{\hat{\omega}}{\hat{\beta}^{2}}, \\
\hat{\beta}^{2}=\frac{U_{2}^{2}-a_{s}^{2}}{a_{s}^{2}}, \\
\hat{\omega}=\frac{\omega}{a_{s}}, \\
\hat{\xi}=\sqrt{\lambda^{2}-\hat{\beta}^{2}}, \\
r^{2}=x_{2}^{2}-\hat{\beta}^{2} y_{2}^{2}, \\
\tanh \theta_{2}=\frac{\hat{\beta}_{2}}{x_{2}}, \\
\lambda=\cot (\beta-\theta) .
\end{gathered}
$$

In deriving (3.16)-(3.18), we have assumed that the harmonic time dependency upstream of the shock is transmitted unchanged through the shock, an assumption that is likely to be accurate provided that $|\epsilon|<<1$ and the wavelength of the disturbance is large compared to the shock thickness. The boundedness argument at the wedge tip $(r=0)$ precludes the $Y_{\nu}(z)$ family of solutions.

If we impose the impermeability condition on the wedge surface, then the $b_{\nu}$ coefficients must all be set to zero; if we demand that the shock remains attached to the wedge 
tip then $d_{0}=0$, and if we assume that there is no transient behaviour (i.e. only the sustained response to the imposed disturbance is present) then $a_{0}=c_{0}=0$ also.

The key useful results are

$$
\begin{aligned}
& \tilde{p}=\frac{\gamma p_{2}}{2 a_{s}} e^{i \omega t-\frac{i \omega U_{2} x_{2}}{U_{2}^{2}-a_{s}^{2}}} \sum_{\nu=0}^{\infty} \hat{k}\left\{2 i a_{\nu}+\frac{U_{2}}{a_{s}} a_{\nu-1}-\frac{U_{2}}{a_{s}} a_{\nu+1}\right\} \cosh \left(\nu \theta_{2}\right) J_{\nu}(\hat{k} r), \\
& \tilde{u}=e^{i \omega t-\frac{i \omega x_{2}}{U_{2}}-\frac{i \omega y_{2} \lambda a_{s}^{2}}{U_{2}\left(U_{2}^{2}-a_{\theta}^{2}\right)}} \sum_{\nu=0}^{\infty} \hat{k}\left\{-i \lambda \frac{a_{s}}{U_{2}} c_{\nu}+\frac{1}{2} \hat{\xi} c_{\nu+1}-\frac{1}{2} \hat{\xi} c_{\nu-1}\right\} J_{\nu}\left(\hat{k} \hat{\xi} y_{2}\right) \\
& -\frac{1}{2} e^{i \omega t-\frac{i \omega U_{2} x_{2}}{U_{2}^{2}-a_{2}^{2}}} \sum_{\nu=0}^{\infty} \hat{k}\left\{2 i \frac{U_{2}}{a_{s}} a_{\nu}+a_{\nu-1}-a_{\nu+1}\right\} \cosh \left(\nu \theta_{2}\right) J_{\nu}(\hat{k} r), \\
& \tilde{v}=e^{i \omega t-\frac{i \omega x_{2}}{U_{2}}-\frac{i \omega y_{2} \lambda a_{s}^{2}}{U_{2}\left(U_{2}^{2}-a_{s}^{2}\right)}} \sum_{\nu=0}^{\infty} \frac{i \hat{k} \hat{\beta}^{2} a_{s}}{U_{2}} c_{\nu} J_{\nu}\left(\hat{k} \hat{\xi} y_{2}\right) \\
& +\frac{1}{2} e^{i \omega t-\frac{i \omega U_{2} x_{2}}{U_{2}^{2}-a_{2}^{2}}} \sum_{\nu=0}^{\infty} \hat{k} \hat{\beta}\left\{a_{\nu-1}+a_{\nu+1}\right\} \sinh \left(\nu \theta_{2}\right) J_{\nu}(\hat{k} r)
\end{aligned}
$$

where $a_{-1}=c_{-1}=d_{-1}=0$. The set of solutions above now involves three sets of coefficients, namely $\left\{a_{\nu}\right\},\left\{c_{\nu}\right\}$, and $\left\{d_{\nu}\right\}$. Although these solutions satisfy the impermeability constraint on $\theta_{2}=0$, we have yet to impose the unsteady Rankine-Hugoniot conditions (McKenzie and Westphal, 1968) on these perturbation terms at the shock, which serve to determine the aforementioned set of coefficients.

The Rankine-Hugoniot conditions on the shock may be written in the following form

$$
\begin{gathered}
u_{2}^{\prime}+\bar{u}_{2} \frac{\tilde{\rho}_{2}}{\rho_{2}}=\left(1-\frac{1}{\rho_{2}}\right) R^{\prime}+\frac{1}{\rho_{2}}\left(\bar{u}_{1} \tilde{\rho}_{1}+u_{1}^{\prime}\right), \\
u_{2}^{\prime}+\frac{1}{2} \bar{u}_{2} \frac{\tilde{\rho}_{2}}{\rho_{2}}+\frac{a_{s}^{2}}{2 \gamma \bar{u}_{2}} \frac{\tilde{p}_{2}}{p_{2}}=u_{1}^{\prime}+\frac{1}{2} \bar{u}_{1} \tilde{\rho}_{1}+\frac{\tilde{p}_{1}}{2 \gamma M_{1}^{2} \bar{u}_{1}}, \\
u_{2}^{\prime}-\frac{a_{s}^{2}}{(\gamma-1) \bar{u}_{2}} \frac{\tilde{\rho}_{2}}{\rho_{2}}+\frac{a_{s}^{2}}{(\gamma-1) \bar{u}_{2}} \frac{\tilde{p}_{2}}{p_{2}}=\left(1-\frac{\bar{u}_{1}}{\bar{u}_{2}}\right) R^{\prime}+\frac{\bar{u}_{1} u_{1}^{\prime}}{\bar{u}_{2}} \\
-\frac{\tilde{\rho}_{1}}{(\gamma-1) M_{1}^{2} \bar{u}_{2}}+\frac{\tilde{p}_{1}}{(\gamma-1) M_{1}^{2} \bar{u}_{2}}, \\
v_{2}^{\prime}=v_{1}^{\prime}+\left(\bar{u}_{1}-\bar{u}_{2}\right) \psi_{\sigma}^{\prime} .
\end{gathered}
$$

Here we have written

$$
R^{\prime}=\bar{v}_{1} \psi_{\sigma}^{\prime}+\psi_{t}^{\prime}
$$


where $\sigma$ denotes distance along the (unperturbed) shock. We have taken $\left(u^{\prime}, v^{\prime}\right)$ to be the velocity perturbations perpendicular and parallel to the shock, respectively, and $(\bar{u}, \bar{v})$ the basic flow perpendicular and parallel to the shock respectively.

The four conditions (3.29)-(3.33) may be combined to eliminate the downstream perturbation density $\rho_{2}^{\prime}$. It is then possible to insert the expressions for $\tilde{p}, \tilde{u}, \tilde{v}, \psi^{\prime}$, all evaluated on the undisturbed shock surface, into these resulting equations to yield the following system of equations at each value of $\nu \geq 0$.

$$
\begin{aligned}
& \cos (\beta-\theta)\left\{\left[-i \lambda \frac{a_{s}}{U_{2}} c_{\nu}+\frac{1}{2} \hat{\xi} c_{\nu+1}-\frac{1}{2} \hat{\xi} c_{\nu-1}\right]\right. \\
& \left.-\frac{1}{2}\left[2 i \frac{U_{2}}{a_{s}} a_{\nu}-a_{\nu+1}+a_{\nu-1}\right] \cosh \left(\nu \theta_{0}\right)\right\} \\
& +\sin (\beta-\theta)\left\{\frac{1}{2} \hat{\beta}\left[a_{\nu-1}+a_{\nu+1}\right] \sinh \left(\nu \theta_{0}\right)+i \hat{\beta}^{2} \frac{a_{s}}{U_{2}} c_{\nu}\right. \\
& \left.-\left(\bar{u}_{1}-\bar{u}_{2}\right)\left[-\frac{i U_{2} \lambda}{a_{s}} d_{\nu}+\frac{1}{2} \hat{\xi}\left(d_{\nu+1}-d_{\nu-1}\right)\right]\right\}=R_{\nu}^{(1)} \\
& \left\{\begin{array}{l}
A_{1} \\
B_{1}
\end{array}\right\}\left\{\operatorname { s i n } ( \beta - \theta ) \left\{\left[-i \lambda \frac{a_{s}}{U_{2}} c_{\nu}+\frac{1}{2} \hat{\xi} c_{\nu+1}-\frac{1}{2} \hat{\xi} c_{\nu-1}\right]\right.\right. \\
& \left.-\frac{1}{2}\left[2 i \frac{U_{2}}{a_{s}} a_{\nu}-a_{\nu+1}+a_{\nu-1}\right] \cosh \left(\nu \theta_{0}\right)\right\} \\
& \left.-\cos (\beta-\theta)\left\{\frac{1}{2} \hat{\beta}\left[a_{\nu-1}+a_{\nu+1}\right] \sinh \left(\nu \theta_{0}\right)+i \hat{\beta}^{2} \frac{a_{s}}{U_{2}} c_{\nu}\right\}\right\} \\
& +\left\{\begin{array}{l}
A_{2} \\
B_{2}
\end{array}\right\} \sin (\beta-\theta)\left\{\bar{v}_{1}\left[-i \lambda \frac{U_{2}}{a_{s}} d_{\nu}+\frac{1}{2} \hat{\xi}\left(d_{\nu+1}-d_{\nu-1}\right)\right]+i \hat{\beta}^{2} a_{s} d_{\nu}\right\} \\
& +\left\{\begin{array}{l}
A_{3} \\
B_{3}
\end{array}\right\}\left\{\frac{1}{2}\left[2 i a_{\nu}+\frac{U_{2}}{a_{s}} a_{\nu-1}-\frac{U_{2}}{a_{s}} a_{\nu+1}\right] \cosh \left(\nu \theta_{0}\right)\right\} \\
& =\left\{\begin{array}{l}
R_{\nu}^{(2)} \\
R_{\nu}^{(3)}
\end{array}\right\} \text {. }
\end{aligned}
$$

Here we have written

$$
\begin{gathered}
\tanh \theta_{0}=\hat{\beta} \tan (\beta-\theta), \\
A_{1}=\frac{1}{2} \\
A_{2}=\frac{1}{2}\left(1-\frac{1}{\rho_{2}}\right), \\
A_{3}=\frac{a_{s}}{2 \bar{u}_{2}}
\end{gathered}
$$




$$
\begin{gathered}
B_{1}=1+\frac{a_{s}^{2}}{(\gamma-1) \bar{u}_{2}^{2}}, \\
B_{2}=-\left(\frac{a_{s}^{2}}{(\gamma-1) \bar{u}_{2}^{2}}-\frac{\bar{u}_{1}}{\bar{u}_{2}}\right)\left(1-\frac{1}{\rho_{2}}\right), \\
B_{3}=\frac{\gamma a_{s}}{(\gamma-1) \bar{u}_{2}} .
\end{gathered}
$$

The determination of $R_{\nu}^{(1)}, R_{\nu}^{(2)}$, and $R_{\nu}^{(3)}$ is routine once the upstream disturbance is prescribed, but for brevity these are omitted. These quantities arise from the forcing caused by the upstream disturbance which is proportional to the exponential (2.7) evaluated at the shock position. This exponential is expanded in terms of the Bessel function by using

$$
\begin{gathered}
\exp \left[i\left(\alpha_{1} \cot \beta+\alpha_{2}\right)(\cos \theta+\lambda \sin \theta) y_{2}\right]=e^{-i \frac{U_{2} \omega \lambda}{V_{2}^{2}-a_{s}^{2}} y_{2}} e^{i \sin \bar{\theta} \hat{k} \tilde{\xi}_{2}} \\
=e^{-i \frac{U_{2} \omega \lambda}{U_{2}^{2}-a_{s}^{2}} y_{2}} \sum_{\nu=0}^{\infty} \frac{e^{i \nu \tilde{\theta}}+(-1)^{\nu} e^{-i \nu \bar{\theta}}}{1+\delta_{\nu, 0}} J_{\nu}\left(\hat{k} \hat{\xi} y_{2}\right)
\end{gathered}
$$

where

$$
\tilde{\theta}=\sin ^{-1}\left\{\frac{\left(\alpha_{1} \cot \beta+\alpha_{2}\right)(\cos \theta+\lambda \sin \theta)+\frac{U_{2} \omega \lambda}{U_{2}^{2}-a_{\theta}^{2}}}{\hat{k} \hat{\xi}}\right\}
$$

and $\delta_{\nu, 0}$ is the Kronecker delta function. Then $a_{\nu+1}, b_{\nu+1}$, and $c_{\nu+1}$ are determined from equations (3.34), (3.35) which are essentially recursive in form.

\section{Analysis of the Series Solution and Far-Downstream Behaviour}

In Figure 1a, we show $\Re\left\{\tilde{p} e^{-i \omega t}\right\}$ (solid line) and $\Im\left\{\tilde{p} e^{-i \omega t}\right\}$ (broken line) both evaluated along the wedge surface for the particular case $M_{1}=5, \theta=25^{\circ}, \alpha_{1}=1$, and $\alpha_{2}=0$, taking the positive sign in (2.8) (the so called "slow mode"). The corresponding shock position $\Re\left\{\psi^{\prime} e^{-i \omega t}\right\}$ (solid line) and $\Im\left\{\psi^{\prime} e^{-i \omega t}\right\}$ (broken line) with distance along the shock, $r_{2}=y_{2}\left(1+\lambda^{2}\right)^{1 / 2}$ is shown in Figure 1b. These results indicate a complex structure to the solution; both quantities show the expected oscillatory behaviour, although the wedgesurface pressure shows indications of amplitude decay as $r_{2} \rightarrow \infty$. Shown in Figure 2 is the response to the so-called "fast mode", i.e. the negative sign in equation (2.8) is used. The wedge-surface pressure is shown in Figure 2a whilst the shock position is shown in Figure $2 \mathrm{~b}$; as before, real components are indicated by solid lines and imaginary components by 
broken lines. The expected oscillatory behaviour is shown, but in this case there is little evidence of amplitude modulation of the wedge-surface pressure. These two examples indicate the importance of ascertaining the correct downstream behaviour of the solution given by the infinite sums (3.26-3.28). Unfortunately, it was found that as $r_{2}$ (or $x_{2}$ ) became large, the computations for $\psi^{\prime}$ (and $\tilde{p}$ ) become extremely sensitive to the number of terms of the series taken (although we stress that all results presented are carefully checked for accuracy, in particular for insensitivity to truncation of the number of terms in the Bessel series (3.16)-(3.18)). Cursory inspection of the coefficients $a_{\nu}, b_{\nu}$, and $d_{\nu}$ indicated that the numerical difficulties described result from these coefficients often becoming extremely large as $\nu \rightarrow \infty$, leading to numerical difficulties associated with the effects of roundoff.

The same general trend of oscillatory, complex behaviour seen in Figures 1 and 2 was also seen in the results of Lasseigne and Hussaini (1992) relating to detonation waves and moving wedges. However, there are two, not unrelated, issues that remain unanswered and affect the physical interpretation of the results. These two issues are: (i) the nature of the coefficients $a_{\nu}, c_{\nu}$ and $d_{\nu}$ as $\nu \rightarrow \infty$ and (ii) the precise nature of the flow far downstream of the shock. We look at these aspects in some detail and discover a number of interesting subtleties.

Superficial inspection of the coefficients indicates general growth as $\nu \rightarrow \infty$. Indeed, this was observed even when the inhomogeneous forcing terms $R_{\nu}^{(1)}, R_{\nu}^{(2)}$, and $R_{\nu}^{(3)}$, were "turned off" after $\nu=1$, in order to mimic a homogeneous system (although in this case it was found that $d_{\nu} \rightarrow 0$ as $\nu \rightarrow \infty$ ). We now therefore consider the homogeneous system for $(3.34),(3.35 \mathrm{a}, \mathrm{b})$ in the limit as $\nu \rightarrow \infty$. This leads to a system of difference equations, and we therefore surmise that the coefficients take on the following form as $\nu \rightarrow \infty$

$$
\begin{aligned}
& c_{\nu} \approx C_{0} K^{\nu}, \\
& d_{\nu} \approx D_{0} K^{\nu},
\end{aligned}
$$

whilst because of the nature of the occurrence of the $a_{\nu}$, we must have

$$
a_{\nu} \approx A_{0} K^{\nu} e^{-\nu \theta_{0}}
$$

where $K$ is an eigenvalue whose precise value is extremely important since it is this value that would be expected to determine the ultimate downstream behaviour of the disturbance 
field. If $|K|>1$, then using the generating function for Bessel functions yields the following result

$$
\sum_{\nu=1}^{\infty} c_{\nu} J_{\nu}(z) \approx \sum_{\nu=1}^{\infty} C_{0} K^{\nu} J_{\nu}(z) \approx C_{0} e^{\frac{1}{2}(K-1 / K) z}, \quad|z| \rightarrow \infty .
$$

This then predicts exponential growth as $|z| \rightarrow \infty$ if $\Re\{z(K-1 / K)\}>0$. Therefore, we proceed to the actual determination of $K$.

Substituting (4.1)-(4.3) into (3.34), (3.35a,b), and discarding inhomogeneous terms, leads to the following three equations to determine $K$ :

$$
\begin{aligned}
& \cos (\beta-\theta)\left\{\left[-i \lambda \frac{a_{s}}{U_{2}}+\frac{1}{2} \hat{\xi} K-\frac{1}{2} \hat{\xi} K^{-1}\right] C_{0}\right. \\
& \left.-\frac{1}{4}\left[2 i \frac{U_{2}}{a_{s}}-e^{-\theta_{0}} K+e^{\theta_{0}} K^{-1}\right] A_{0}\right\} \\
& +\sin (\beta-\theta)\left\{\frac{1}{4} \hat{\beta}\left[e^{-\theta_{0}} K+e^{\theta_{0}} K^{-1}\right] A_{0}\right. \\
& \left.+i \hat{\beta}^{2} \frac{a_{s}}{U_{2}} C_{0}-\left(\bar{u}_{1}-\bar{u}_{2}\right)\left[-\frac{i U_{2} \lambda}{a_{s}}+\frac{1}{2} \hat{\xi} K-\frac{1}{2} \hat{\xi} K^{-1}\right] D_{0}\right\}=0, \\
& \left\{\begin{array}{l}
A_{1} \\
B_{1}
\end{array}\right\}\left\{\operatorname { s i n } ( \beta - \theta ) \left\{\left[-i \lambda \frac{a_{s}}{U_{2}}+\frac{1}{2} \hat{\xi} K-\frac{1}{2} \hat{\xi} K^{-1}\right] C_{0}\right.\right. \\
& -\frac{1}{4}\left[2 i \frac{U_{2}}{a_{s}}-K e^{-\theta_{0}}+K^{-1} e^{\theta_{0}}\right] A_{0} \\
& \left.-\cos (\beta-\theta)\left\{\frac{1}{4} \hat{\beta}\left[K e^{-\theta_{0}}+K^{-1} e^{\theta_{0}}\right] A_{0}+i \hat{\beta}^{2} \frac{a_{s}}{U_{2}} C_{0}\right\}\right\} \\
& \left.+\left\{\begin{array}{l}
A_{2} \\
B_{2}
\end{array}\right\} \sin (\beta-\theta)\left\{\bar{v}_{1}\left[-\frac{i U_{2} \lambda}{a_{s}}+\frac{1}{2} \hat{\xi}\left(K-K^{-1}\right)\right]+i \hat{\beta}^{2} a_{s}\right] D_{0}\right\} \\
& +\left\{\begin{array}{l}
A_{3} \\
B_{3}
\end{array}\right\}\left\{\frac{1}{4}\left[2 i+\frac{U_{2}}{a_{s}} K^{-1} e^{\theta_{0}}-\frac{U_{2}}{a_{s}} K e^{-\theta_{0}}\right] A_{0}\right\} \\
& =0 \text {. }
\end{aligned}
$$

This system may conveniently be expressed in the form of a sixth-order linear generalized eigenvalue problem by writing

$$
\begin{aligned}
& \hat{A}_{0}=K A_{0}, \\
& \hat{C}_{0}=K C_{0}, \\
& \hat{D}_{0}=K D_{0},
\end{aligned}
$$


and solving for the eigenvector $\left\{A_{0}, \hat{A}_{0}, C_{0}, \hat{C}_{0}, D_{0}, \hat{D}_{0}\right\}$. This was accomplished numerically and yielded the following general trends: (i) Of the six roots for $K$ of the eigenvalue problem, four were imaginary, and the remaining two were complex twins $K=K_{0}$ and $K=-$ c.c. $\left\{K_{0}\right\}$ (indeed, the nature of these roots can be confirmed by inspection of the system $(4.5),(4.6 \mathrm{a}, \mathrm{b})$; (ii) the imaginary roots all had $0<|K|<1$; (iii) the complex pair of roots had $|K|>1$. This last point strongly suggests the flow is structurally unstable since there exists homogeneous solutions for $\phi$ and $E$ which grow exponentially as the distance from the wedge tip increases; however, further detailed analysis given later shows this to be untrue.

Returning now to consider the nature of the imaginary roots, Figure 3 shows the variation of these roots with wedge angle $\theta$ at $M_{1}=1.5,3$, and 5 . In addition to the trends outlined above, the roots have the features that $K \rightarrow 0$ as $\theta \rightarrow 0$, whilst as $\theta \rightarrow \theta_{m}$, $|K| \rightarrow 1$, where $\theta_{m}$ is the limit of the envelope of supersonic flow downstream of the shock. This then, in some way connects with the results of previous research on the stability of the strong shock solution. We now consider the nature of the complex roots. Fortunately, it is possible to obtain precise analytic information on these roots aided by the observation that the eigenvectors from the computation reveal that $D_{0}=\hat{D}_{0}=0$ (to within machine precision). If we use this result, then the groupings shown in $(4.5),(4.6)$ would suggest that an eigensolution is possible if the following three equations are simultaneously satisfied:

$$
\begin{gathered}
{\left[-i \lambda \frac{a_{s}}{U_{2}}+\frac{1}{2} \hat{\xi} K-\frac{1}{2} \hat{\xi} K^{-1}\right] C_{0}-\frac{1}{4}\left[2 i \frac{U_{2}}{a_{s}}-e^{-\theta_{0}} K+e^{\theta_{0}} K^{-1}\right] A_{0}=0} \\
\frac{1}{4}\left[e^{-\theta_{0}} K+e^{\theta_{0}} K^{-1}\right] A_{0}+i \hat{\beta} \frac{a_{s}}{U_{2}} C_{0}=0 \\
2 i-\frac{U_{2}}{a_{s}} e^{-\theta_{0}} K+\frac{U_{2}}{a_{s}} e^{\theta_{0}} K^{-1}=0 .
\end{gathered}
$$

We find this is indeed possible and the solution is

$$
\begin{gathered}
K=\frac{e^{\theta_{0}}}{U_{2}}\left\{i a_{s} \pm\left[U_{2}^{2}-a_{s}^{2}\right]^{1 / 2}\right\}, \\
C_{0}= \pm i A_{0} / 2 .
\end{gathered}
$$


The value of $K$, given by (4.11) has $|K|=e^{\theta_{0}}>1$, suggesting that the flow is always structurally unstable in the sense that disturbances will always grow downstream. However, it turns out that in fact this homogeneous solution represents a structurally stable flow. This stability is demonstrated by defining the following functions which arise naturally as a result of the analysis above

$$
\tilde{E}=C_{0} e^{i \omega t-\frac{i \omega x_{2}}{U_{2}}-\frac{i \omega y_{2} \lambda a_{s}^{2}}{U_{2}\left(U_{2}^{2}-a_{\theta}^{2}\right)}} \sum_{\nu=-\infty}^{\infty} K^{\nu} J_{\nu}\left(\hat{k} \hat{\xi} y_{2}\right),
$$

together with

$$
\tilde{\phi}=A_{0} e^{i \omega t-\frac{i \omega U_{2} x_{2}}{U_{2}^{2}-a_{a}^{2}}} \sum_{\nu=-\infty}^{\infty}\left\{K e^{\theta_{2}-\theta_{0}}\right\}^{\nu} J_{\nu}(\hat{k} r)
$$

$\tilde{E}$ and $\tilde{\phi}$ are both harmonic and related through the Cauchy-Riemann equations, with

$$
\tilde{\phi}_{x_{2}}=-\tilde{E}_{y_{2}}, \quad \tilde{\phi}_{y_{2}}=\tilde{E}_{x_{2}} .
$$

As a direct consequence of (4.15), the exponential growth of the two components to this homogeneous solution cancel each other when computing physical quantities such as the pressure $\tilde{p}$ or the velocity $(\tilde{u}, \tilde{v})$, and therefore, the flow calculated from the summations (3.26)-(3.28) is bounded for all values of $\left(x_{2}, y_{2}\right)$.

Although, the growing homogeneous solution for the coefficients does not lead to a physically unbounded solution, due to exact cancellations when calculating physical quantities, their presence greatly complicates the computation of the summations (3.26)-(3.28) for calculating the flows since the relevant physical quantities result from the remainder of summing three or more, numerically very large quantities, to yield a much smaller quantity. The numerical difficulties in obtaining accurate solutions associated with this approach are obvious. We note (again) that all results presented have been carefully checked, to account for this phenomenon.

The final question that arises is the nature of the downstream response to sustained acoustic waves, entropy waves, or vorticity waves. We may expect that the solution downstream will remain oscillatory, with no amplitude decay, although this is not always the case. The different downstream behaviours of the acoustic disturbances can be understood by firstly examining the solution in the absence of the wedge (see McKenzie \& Westphal 
1968). Since the frequency of the disturbance downstream of the shock is fixed by the frequency of the disturbance upstream of the shock and the wavenumber of the disturbance tangential to the shock is also fixed by the deflection of the shock produced by the upstream disturbance, there is a finite range of wavenumbers tangential to the shock that exclude the existence of plane acoustic waves which propagate downstream. If the imposed tangential wavenumber lies within this range, then the acoustic disturbance behind the shock (in the absence of the wedge) exhibits exponential decay in a direction normal to the shock. If the tangential wavenumber lies outside of this range, then a plane wave propagates at an angle $\theta_{p}$ to the shock. This angle and the range of excluded tangential wavenumbers depend upon the relative orientation of the shock and the upstream base-flow and disturbance conditions.

The presence of the wedge changes the nature of these solutions drastically. In the case of the tangential wavenumber being within the excluded range, an acoustic field is generated that decays algebraically (rather than exponentially) owing to the requirement that the shock remain attached to the apex and the requirement that disturbances have zero normal velocity at the wedge surface. If the tangential wavenumber is outside of the excluded range, then the solution in the presence of the wedge exhibits two types of behaviour, dependent upon the angle of propagation of an acoustic disturbance in the absence of the wedge. If the angle of propagation $\theta_{p}$ is greater than the angle between the shock and the wedge $\beta-\theta$, then the pressure disturbances generated at the shock would intersect with and reflect from the wedge surface. Thus, there would be a non-decaying pressure field everywhere between the shock and the wedge surface with a superimposed algebraically decaying pressure field owing to the requirement of an attached shock and zero velocity normal to the wedge surface. If the angle of propagation $\theta_{p}$ is less than the angle between the shock and the wedge surface, then the pressure field is divided into two regions by a ray emanating from the apex and parallel to the direction of propagation of the pressure disturbance in the absence of the wedge. Between the shock and this ray there is a non-decaying pressure field, with the aforementioned superimposed non-decaying component. Between the ray and the wedge surface the pressure field is algebraically decaying and there is a component with sustained oscillations. This is in contrast to the 
solution in the absence of the wedge and is explained by the presence of the wedge that serves to terminate the shock (which is assumed of infinite extent in both directions in the absence of the wedge), and there are no pressure disturbances generated by the shock to fill this region. We will refer to this region as the shadow region.

The above mentioned downstream limits can be mathematically understood by again considering the behaviour of the coefficients $a_{\nu}, c_{\nu}$, and $d_{\nu}$ as $\nu \rightarrow \infty$ when the inhomogeneous terms $R_{\nu}^{(1)}, R_{\nu}^{(2)}$,and $R_{\nu}^{(3)}$ are included. For this, the nature of $\tilde{\theta}$ in (3.44) is important. If $\tilde{\theta}$ is real, then the wavenumber of the imposed disturbances lies within the excluded range derived from the theory of McKenzie and Westphal (1968), the coefficients within the summation (3.43) neither grow nor decay, and the combination of coefficients within the bracket in equation (3.26) all decay at a rate proportional to $e^{-\nu \theta_{0}}$. Thus when reconstructing the pressure along any ray $\theta_{2}=$ constant, algebraic decay is found since $\theta_{2}$ must lie on the interval $\left(0, \theta_{0}\right)$ with zero corresponding to the wedge surface and $\theta_{0}$ corresponding to the shock. For $\theta_{2}=\theta_{0}$ the bracketed combination of coefficients does not decay leading to the appropriate oscillatory behaviour necessary to match conditions at the shock. If $\tilde{\theta}$ is complex, then the condition

$$
\left|\frac{\left(\alpha_{1} \cot \beta+\alpha_{2}\right)(\cos \theta+\lambda \sin \theta)+\frac{U_{2} \omega \lambda}{U_{2}^{2}-a_{s}^{2}}}{\hat{k} \hat{\xi}}\right|>1,
$$

is satisfied. We also note that for equation (3.43) to be satisfied then $\sin \tilde{\theta}$ must remain real, which requires that

$$
\tilde{\theta}= \pm \pi / 2-i \tilde{\theta}_{i}
$$

where

$$
\tilde{\theta}_{i}=\cosh ^{-1}\left\{ \pm \frac{\left(\alpha_{1} \cot \beta+\alpha_{2}\right)(\cos \theta+\lambda \sin \theta)+\frac{U_{2} \omega \lambda}{U_{2}^{2}-a_{2}^{2}}}{\hat{k} \hat{\xi}}\right\} .
$$

Notice $\tilde{\theta}_{i}$ is related to the angle between the direction of the shock and the direction of the acoustic disturbance $\theta_{p}$ through $\tanh \tilde{\theta}_{i}=\hat{\beta} \tan \theta_{p}$. For imaginary values of $\tilde{\theta}$ the coefficients of (3.43) behave as $\left( \pm i e^{\tilde{\theta}_{i}}\right)^{\nu}$, as $\nu \rightarrow \infty$, which increase exponentially in magnitude as do the corresponding coefficients $R_{\nu}^{(1)}, R_{\nu}^{(2)}$, and $R_{\nu}^{(3)}$. If $\tilde{\theta}_{i}$ is greater than $\theta_{0}$, then the generated or transmitted acoustic waves intersect the wedge surface. The physical consequence of this intersection is seen by considering the bracketed combination of coefficients 
in (3.26) along with the factor $\cosh \left(\nu \theta_{2}\right)$. The product grows exponentially and alternates between real and imaginary. The relation (4.4) shows that there is a sustained oscillatory solution of the pressure for all $x_{2}$ and $y_{2}$. For $0<\tilde{\theta}_{i}<\theta_{0}$, the aforementioned product of coefficients is exponentially growing when $\theta_{2}>\tilde{\theta}_{i}$ which shows that there is a region of sustained oscillatory behaviour of the pressure field near the shock, and the aforementioned product of coefficients is exponentially decaying for $\theta_{2}<\tilde{\theta}_{i}$ which shows that there is a region of algebraic decay of the pressure field near the wedge surface and that there are no sustained oscillations near the surface. This overall behaviour of the pressure field has been verified by direct computation of the summation (3.26) along rays $\theta_{2}=$ constant for various cases.

With the previous analysis in mind and restating that one goal of this analysis is to discover how these solutions might affect the boundary layer receptivity problem, we focus attention on the pressure evaluated along the wedge which generally may naturally be decomposed into four parts

$$
\begin{aligned}
e^{-i \omega t} \tilde{p} & =P_{0} e^{-\frac{i \omega U_{2} x_{2}}{U_{2}^{2}-a_{s}^{2}}+i \hat{k} \cosh \left(\tilde{\theta}_{i}-\theta_{0}\right) x_{2}} \\
& +P_{1} x_{2}^{-1 / 2} e^{-\left(\frac{i \omega x_{2}}{U_{2}-a_{o}}-i \frac{\pi}{4}\right)} \\
& +P_{2} x_{2}^{-1 / 2} e^{-\left(\frac{i \omega x_{2}}{U_{2}+a_{s}}-i \frac{\pi}{4}\right)}+Q\left(x_{2}\right) .
\end{aligned}
$$

The first term on the right-hand-side is non-zero only when $\tilde{\theta}_{i}>\theta_{0}$ and is evaluated numerically by considering the large $\nu$ behaviour the coefficients $\left\{a_{v}\right\}$. This term is converted to an infinite sum of Bessel functions and then subtracted from the left-hand-side where the pressure has the representation given by the summation (3.26). The Fourier Sine Transform of the resulting difference is evaluated and the strengths of the inverse square root singularities provide the amplitudes $P_{1}$ and $P_{2}$ for the second and third terms. Physically, the first term represents the transmitted acoustic wave (or generated acoustic wave if the imposed disturbance is an entropy or a vorticity wave) when it intersects the wedge surface, and the second and third term are the far-downstream behaviour of slow and fast moving acoustic waves associated with reflections off the wedge surface and regeneration of new acoustic waves at the shock, as well as new acoustic waves generated by the requirement that the shock remains attached to the wedge tip. The first three terms are 
the leading terms in the limit $x_{2} \rightarrow \infty$, and the fourth term decays faster than $x_{2}^{-1 / 2}$ and is considered to be the local field. The Fourier spectrum of the fourth term is important since it represents the generation of all wavelengths by the response of the attached shock to a single wavelength input; the spectrum of the local field is bounded for all wavenumbers since the local field decays relatively quickly as $x_{2} \rightarrow \infty$.

If $\tilde{\theta}_{i}<\theta_{0}$ then the far-downstream behaviour of the pressure along the surface changes. The wavenumber of the imposed disturbance is outside of the allowable wavenumbers for propagating plane waves or the generated or transmitted plane-wave pressure disturbance does not intersect the wedge, and it is found that $P_{0}$ is zero. The amplitudes $P_{1}$ and $P_{2}$ are non-zero and therefore the pressure has algebraic decay far downstream.

The vorticity, found by computing $\Omega=\nabla^{2} E$, can be similarly decomposed into four parts if $\tilde{\theta}_{i}>0$

$$
\begin{aligned}
e^{-i \omega t+\frac{i \omega x_{2}}{U_{2}}} \Omega & =e^{-\frac{i \omega a_{2}^{2} \lambda_{y_{2}}}{U_{2}\left(U_{2}^{2}-a_{\xi}^{2}\right)}}\left\{\Omega_{0} e^{i \hat{k} \cosh \bar{\theta}_{i} y_{2}}\right. \\
& +\Omega_{1} y_{2}^{-1 / 2} e^{-\left(i \hat{k} \hat{\xi} y_{2}-i \frac{\pi}{4}\right)} \\
& \left.+\Omega_{2} y_{2}^{-1 / 2} e^{i \hat{k} \hat{\xi}_{2}+i \frac{\pi}{4}}\right\}+q\left(y_{2}\right)
\end{aligned}
$$

where we have assumed that the calculations take place far enough downstream in $x_{2}$ such that the behaviour represented by $(4.21)$ is an accurate expansion valid for large $y_{2}$. The first term represents a generated or transmitted vorticity wave and the next two terms represent additional vorticity generated by the moving shock as a result of requiring that the shock remain attached to the wedge apex and zero normal velocity on the surface. That is, the algebraically decaying component of the pressure field generated by the presence of the wedge interacts with the shock wave through the Rankine-Hugoniot relations which indicate that the shock must be distorted by this component of the pressure field, and hence algebraically decaying vorticity modes represented by $\Omega_{1}$ and $\Omega_{2}$ are generated by the curvature of the shock. A numerical subtlety exists when trying to calculate the decomposition (4.21) when $\tilde{\theta}_{i}>0$. The coefficient of the vorticity depends on cancellations involving five very large terms (for the pressure decomposition there were only three very large terms); thus, it is not possible to accurately determine $\Omega_{0}$ for some cases. If $\tilde{\theta}_{i}=0$, then it appears to be not possible to calculate a decomposition of the vorticity into the form 
given in (4.21). The coefficients of the vorticity expansion neither grow nor decay making the numerical determination of $\Omega_{0}$ difficult if not impossible. In the absence of the wedge, a single wavelength vorticity mode would still exist for this case, and the pressure field would be exponentially decaying. With the wedge present, there exists a small algebraic component of the pressure field.

The results relating to the decompositions given in (4.20) and (4.21) are given in the next section.

\section{Results}

The response of the flow downstream of the shock is generally complicated, although the analysis of the previous section does enable us to quantify the response, at least in the far downstream limit. To this end, we find it useful to consider the sine-transform of $Q\left(x_{2}\right)$, that is

$$
Q^{*}=\int_{0}^{\infty} Q\left(x_{2}\right) \sin \left(\mu x_{2}\right) d x_{2}
$$

Fortunately, this quadrature can be carried out semi-analytically using the following sinetransform

$$
\begin{aligned}
\int_{0}^{\infty} \sin \left(\mu x_{2}\right) e^{i q x_{2}} J_{\nu}\left(\hat{k} x_{2}\right) d x_{2}= & \frac{1}{2 i \hat{k}^{\nu}} \frac{\left\{\sqrt{\hat{k}^{2}-(\mu+q)^{2}}+i(\mu+q)\right\}^{\nu}}{\sqrt{\hat{k}^{2}-(\mu+q)^{2}}} \\
& -\frac{1}{2 i \hat{k}^{\nu}} \frac{\left\{\sqrt{\hat{k}^{2}-(\mu-q)^{2}}-i(\mu-q)\right\}^{\nu}}{\sqrt{\hat{k}^{2}-(\mu-q)^{2}}} .
\end{aligned}
$$

The inverse square-root singularities lead to the second and third terms of (4.20) and the evaluation of (5.1) involves a summation of terms derived from (5.2) with coefficients determined numerically. The results of this process are shown graphically in Figures 4-8 where $\Re\left\{Q^{*}\right\}$ is denoted by solid lines, whilst $\Im\left\{Q^{*}\right\}$ is denoted by broken lines.

The spectrum $Q^{*}$ for the input conditions $M_{1}=5, \alpha_{1}=1, \alpha_{2}=0$ and $\theta=25^{\circ}$ (slow mode) is shown in Figure 4. For this set of input conditions, inequality (4.17) is not satisfied and therefore $P_{0}$ in (4.20) is zero. This spectrum is bounded for all wavenumbers 
but has square-root behaviour at $\mu=\frac{\omega}{U_{2} \pm a_{\mathrm{s}}}$, the wavenumber locations of the slow and fast acoustic disturbances behind the shock. This square-root behaviour remains after subtracting the inverse square-root singularity to form the second and third terms of (4.20). For wavenumbers between these two points, a plane wave with the fixed time frequency $\omega$ would have to travel faster than the sound speed relative to the mean flow, and so these represent pressure disturbances that decay exponentially in $x_{2}$. The local field is comprised of a Fourier integral over both classes of disturbance. It should be noted that the large wavenumber disturbances are "slow modes" whilst the small wavenumber disturbances are "fast modes". The importance of $Q^{*}$ is that it demonstrates that far downstream the energy density of the acoustic response is spread over all wavenumbers and is not concentrated at an isolated wavenumber as is the energy density of the acoustic mode in the absence of the wedge. In particular, the energy density is significant at large wavenumbers, i.e small wavelengths, and these disturbances generated by the shock-body interaction might lead to receptivity as they interact with a growing boundary layer on the wedge surface.

Figure 5 shows the spectrum for the fast mode with the same input conditions as in Figure 4. For the fast mode, inequality (4.17) is satisfied so that $P_{0}$ is non-zero. To indicate the presence of this transmitted plane wave, a circle has been added to Figure 5 at the wavenumber location of the transmitted wave. It should be noted that this wave number location is not quite at the same wavenumber as the singularity of the Fourier transform which leads to the second and third terms of the expansion. Figure 4 and Figure 5 appear to be quantitatively similar, although a shift to smaller wavenumbers is forced by the changed frequency of the imposed disturbance, a result of choosing the opposite sign in equation (2.8).

In Figure 6, the spectrum of a fast mode with larger incoming Mach number is shown. The input conditions are $M_{1}=10, \alpha_{1}=1, \alpha_{2}=0$ and $\theta=25^{\circ}$ (slow mode). This set of input conditions also falls within the envelope of flows which lead to sustained downstream acoustic waves. It is seen that the energy density of the local field has a strong Mach number dependence, as do the amplitudes of the first three terms of (4.20) which will be explored in Figures 9-12. Figures 7 and 8 show the spectra for the local pressure field $Q^{*}$, in response to an incoming entropy disturbance (2.13-2.16) and to an 
incoming vorticity disturbance (2.9-2.12). The input conditions are the same as in Figure 4 (except for Figure 8, for which we have taken $\alpha_{1}=0$ and $\alpha_{2}=1$ ). There is a qualitative difference between the local field response to acoustic disturbances and the local field response to these non-acoustic inputs. The spectra in Figures 7 and 8 are considerably more peaked near the fast-mode singularity with the response near the slow-mode singularity not nearly as strong. For the response to both the fast and slow incoming acoustic modes, the spectra near both singularities were broad with the strength of the slow-mode singularity as strong or stronger than the fast-mode singularity. Perhaps this is a result of the incoming entropy disturbance having zero pressure and velocity components, the incoming vorticity wave having zero pressure and density components, whilst all components of the incoming acoustic disturbances are non-zero, thus leading to a much more complicated interaction with the attached shock (especially near the tip of the wedge).

The amplitude of the transmitted or generated (sustained) acoustic disturbance $P_{0}$, the amplitude of the algebraically decaying slow mode $P_{1}$, and the amplitude of the algebraically decaying fast mode $P_{2}$ are shown graphically in Figures 9-12. It can be shown that $P_{0}$ and $P_{2}$ are real quantities whilst $P_{1}$ is strictly imaginary. $\Re\left\{P_{0}\right\}$ is denoted as a solid line, $\Im\left\{P_{1}\right\}$ is denoted as a line with small dashes and $\Re\left\{P_{2}\right\}$ is denoted as a line with large dashes.

Figure 9a shows the response for the an impinging slow acoustic mode. Over a large range of Mach numbers of the incoming flow $M_{1}$, there exists no downstream sustained acoustic waves, i.e $P_{0}=0$; however, both of the algebraically decaying modes are non-zero, and the amplitudes increase considerably as the Mach number increases. As mentioned in the introduction, this is significant since in the absence of the wedge the theory (as verified by numerical computations) predicts that the acoustic response will be exponentially decaying, but by requiring that the shock remains attached to the wedge tip, it is seen that the response decays like the inverse square root (together with a local field which also does not decay exponentially). Figure $9 \mathrm{~b}$ shows the details of the transition region (in Mach number) between the decaying pressure response and the sustained pressure response. The amplitudes of $P_{1}$ and $P_{2}$ jump dramatically when $P_{0}$ is non-zero. Physically, this implies that there are two parts to the algebraically decaying modes. The first part is a result of 
the requirement that the shock remain attached as seen when $P_{0}=0$; the second part is a direct result of the sustained acoustic disturbance reflecting off the wedge surface and interacting with the shock to generate more acoustic disturbances. As $M_{1}$ decreases, the critical wedge angle (above which there is no mean flow solution) decreases until it reaches the fixed wedge angle of Figures 9-12 (i.e. $\theta=25^{\circ}$ ). The amplitude of the transmitted wave remains finite in this limit; however $P_{1}$ and $P_{2}$ increase sharply in magnitude, this implies that the solution is becoming increasingly unstable as the critical angle is approached (as would be expected). In Figures 10-12, the response to an incoming fast acoustic wave, to an incoming entropy wave and to an incoming vorticity wave, respectively, are shown; in all cases $\theta=25^{\circ}, M_{1}=5, \alpha_{1}=1$, and $\alpha_{2}=0$ except in the case of the vorticity mode (Figure 12) where $\alpha_{2}=1$ and $\alpha_{1}=0$. There is a sustained acoustic disturbance for these three cases, and correspondingly, the amplitudes of $P_{1}$ and $P_{2}$ are relatively large since as mentioned in the discussion of Figure 9 these two modes have components related to the reflection of the acoustic waves from the wedge surface. All three figures demonstrate the same behaviour as Figure 9 when the critical angle is approached - the amplitude of the sustained mode remains finite whilst the other modes grow. For the responses to the impinging acoustic disturbance (Figure 10) and to the impinging entropy disturbance (Figure 11 ), the algebraically decaying fast-mode is considerably larger than the slow-mode with the relative importance of the slow-mode being greater for the response to the entropy wave. Comparing the spectra shown in Figures 5 and Figure 7, the energy of the slow mode is seen to be in the local fields with relatively more energy for the slow-mode in the response to the impinging acoustic wave. In the response to the incoming vorticity disturbance (Figure 12), the amplitude of the slow mode $P_{1}$ is much larger than the amplitude of the fast-mode $P_{2}$. Comparing with Figure 8 , this is consistent with the relative absence of energy in the slow-mode of the local field.

For completeness, Figure 13 and Figure 14 show the result of a decomposition of the vorticity into the form given by (4.21). Figure 13 represents a response to an incoming fast acoustic wave with the same conditions as Figure 10, but with a wedge angle of only $20^{\circ}$. The change of wedge angle is due to the numerical difficulties of calculating $\Omega_{0}$ already mentioned. Figure 14 shows the response to an incoming entropy wave. General 
conclusions about the decomposition are difficult to obtain (again related to the numerical difficulties); however, we do note the relative insensitivity to the Mach number of the coefficients except near the critical angle where one of the algebraically decaying components becomes quite large. Again, this probably relates directly to the stability of the entire solution as the critical angle is approached.

\section{Conclusions}

We have considered the interaction between the shock wave attached to the wedge and freestream disturbances. In order to quantify the results, the freestream disturbances were taken to be fixed-frequency, single-wavelength, plane-wave disturbances of acoustic type, the vorticity type, or entropy type. The behaviour of the flow field downstream of the shock in the shock-wedge combination was compared with the behaviour of the downstream flow in the absence of the wedge. After rationalizing a number of mathematical and computational subtleties, we were able to show precisely how the presence of the wedge affects the interaction between a shock and a free stream disturbance. We specifically focused on the pressure disturbance along the wedge surface as the analysis of these disturbances directly relates to boundary-layer receptivity in the shock-wedge configuration and we also studied the vorticity generated by the presence of the wedge as this analysis relates to enhanced mixing by the vorticity production of a moving shock.

The most significant differences in the interaction produced by the presence of the wedge are the presence of an acoustic and vorticity field which decays algebraically in addition to sustained oscillations produced by the incoming disturbance. It has been shown that this field is comprised of two parts: one produced by the requirement that the shock remain attached to the apex of the wedge and the other produced by reflections of acoustic waves from the wedge surface. 


\section{References}

Anyiwo, J.C., and Bushnell, D.M. 1982 Turbulence Amplification in Shock wave/ boundary-layer interaction, AIAA J. $\underline{20}, 893$.

Carrier, G.F. 1949a, The Oscillatory wedge in a supersonic stream. J. Aero. Sci. 16, 150.

Carrier, G.F. 1949b, On the stability of the supersonic flow past a wedge. Q. Appl. Math. $\underline{6}, 356$.

Chang, C.L., Malik, M.R. and Hussaini, M.Y., 1990, Effects of shock on the stability of hypersonic boundary layers. AIAA paper 90-1448.

Cowley, S.J. and Hall, P. 1988, On the stability of hypersonic flow past a wedge, ICASE report 88-72.

Henderson, L.F. and Atkinson, J.D. 1970, Multi-valued solutions of steady-state supersonic flow. Part I. Linear Analysis. J. Fluid Mech. $\underline{75}, 751$.

Hui, W.H. 1969, Stability of oscillating wedges and Caret wings in hypersonic and supersonic flows. AIAA J. $\underline{7}, 1524$.

Hussaini, M.Y., Collier, F. and Bushnell, D.M. 1985, Turbulence alteration due to shock motion. ICIAM Symposium, Turbulent Shear-layer/Shock wave Interaction. ed. J. Delany, Springer, New York, p.371.

Jackson, T.L., Kapila, A.K. and Hussaini, M.Y. 1990, Convection of a pattern of vorticity through a reacting shock wave. Phys. Fluids, $\underline{\mathrm{A} 2}, 1260$.

Kumar, A., Bushnell, D.M., and Hussaini, M.Y. 1989, Mixing augmentation technique for hypervelocity scramjet. J. Prop. and Power $\underline{5}, 514$.

Lasseigne, D.G. and Hussaini, M.Y. 1991, Disturbance pattern associated with an oblique detonation wave attached to a wedge, submitted to Phys. Fluids $\underline{A}$.

Levinson, J.L. 1945, L'etude de la stabilite des courant supersonique de gas en relation avec on double solution de la théorie des ondes de choc. Prikl. Mat. Mekh. 9 , 151.

Liepmann, H.W. and Roshko,A. 1957, Elements of gas dynamics. John Wiley and Sons.

McKenzie,J.F. and Westphal, K.O. 1968, Interaction of linear waves with oblique shock waves. Phys. Fluids. 11, 2310. 
Meadows, K. R., Kumar, A., and Hussaini,M.Y. 1991, A Computational Study on the Interaction Between a Vortex and a Shock Wave. AIAA J. $\underline{29}, 174$

Moore, F.K. 1954, Unsteady oblique interaction of a shock wave with a plane disturbance. NACA report 1165 .

Petrov, G.V., 1984, Stability of a thin viscous layer on a wedge in hypersonic flow of a perfect gas, in Laminar Turbulent Transition, ed. V.V. Kozlov, Proc. 2nd IUTAM Symp., Springer.

Ribner,H.S. 1954, Convection of a pattern of vorticity through a shock wave, NACA report 1164.

Rusanov, V.V. and Sharakshange, A.A. 1980, On the non-uniqueness of the solution of the problem on steady flow about the plane wedge and circular cone, Computers and Fluids $\underline{7}, 243$.

Salas, M.D. and Morgan, B.D. 1982, On the instability of shock waves attached to wedges and cones, Proc. AIAA 20th Aerospace Science meeting.

Stewartson, K. 1964, Compressible boundary layers, Oxford U.P.

Van Dyke, M.D. 1953, On supersonic flow past an oscillating wedge, Quart. Appl. Math. 11,360 . 


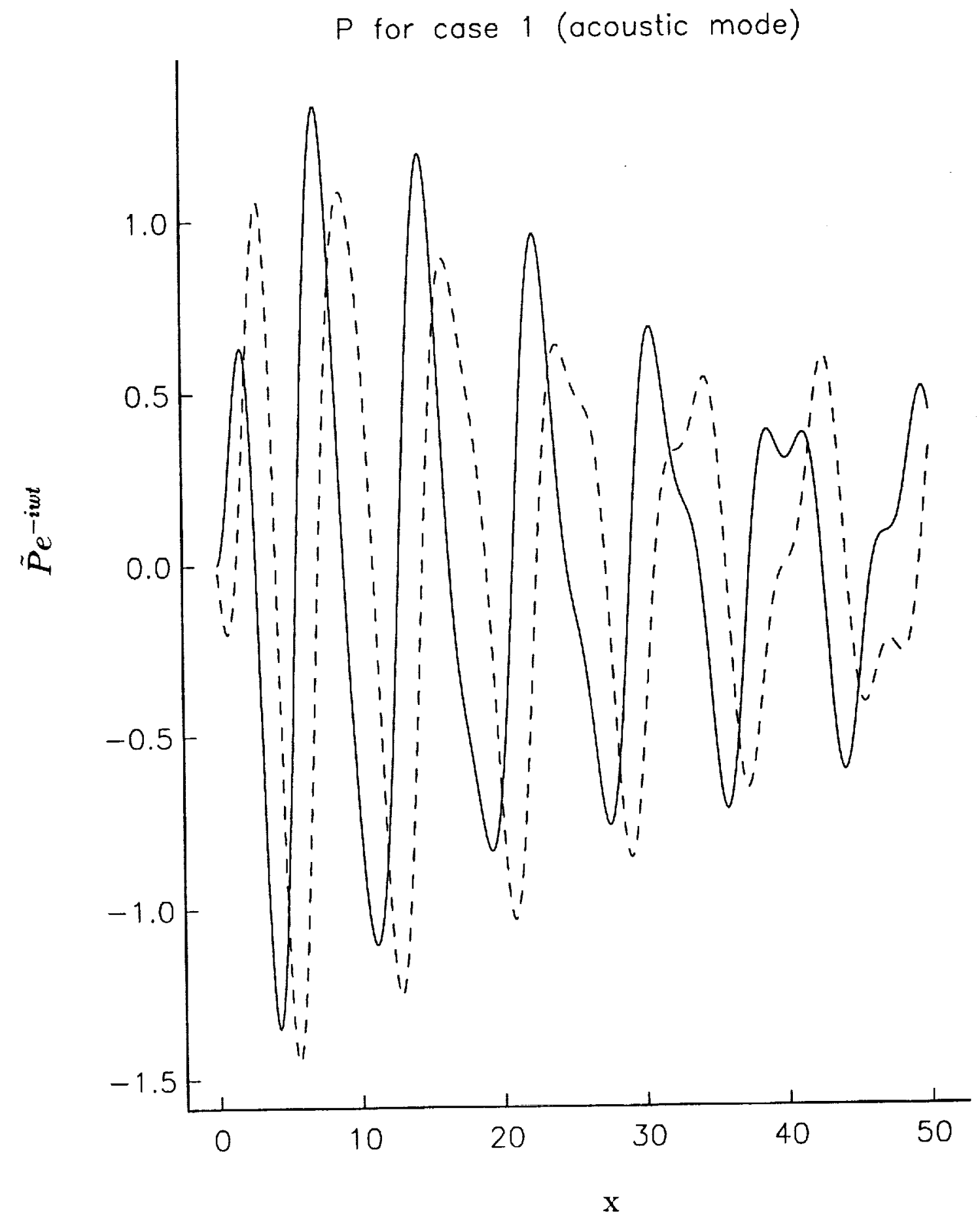

Fig.1a Wall pressure distribution for upstream slow acoustic mode, $M_{1}=5, \theta=25^{\circ}, \alpha_{1}=1$, $\alpha_{2}=0$. 


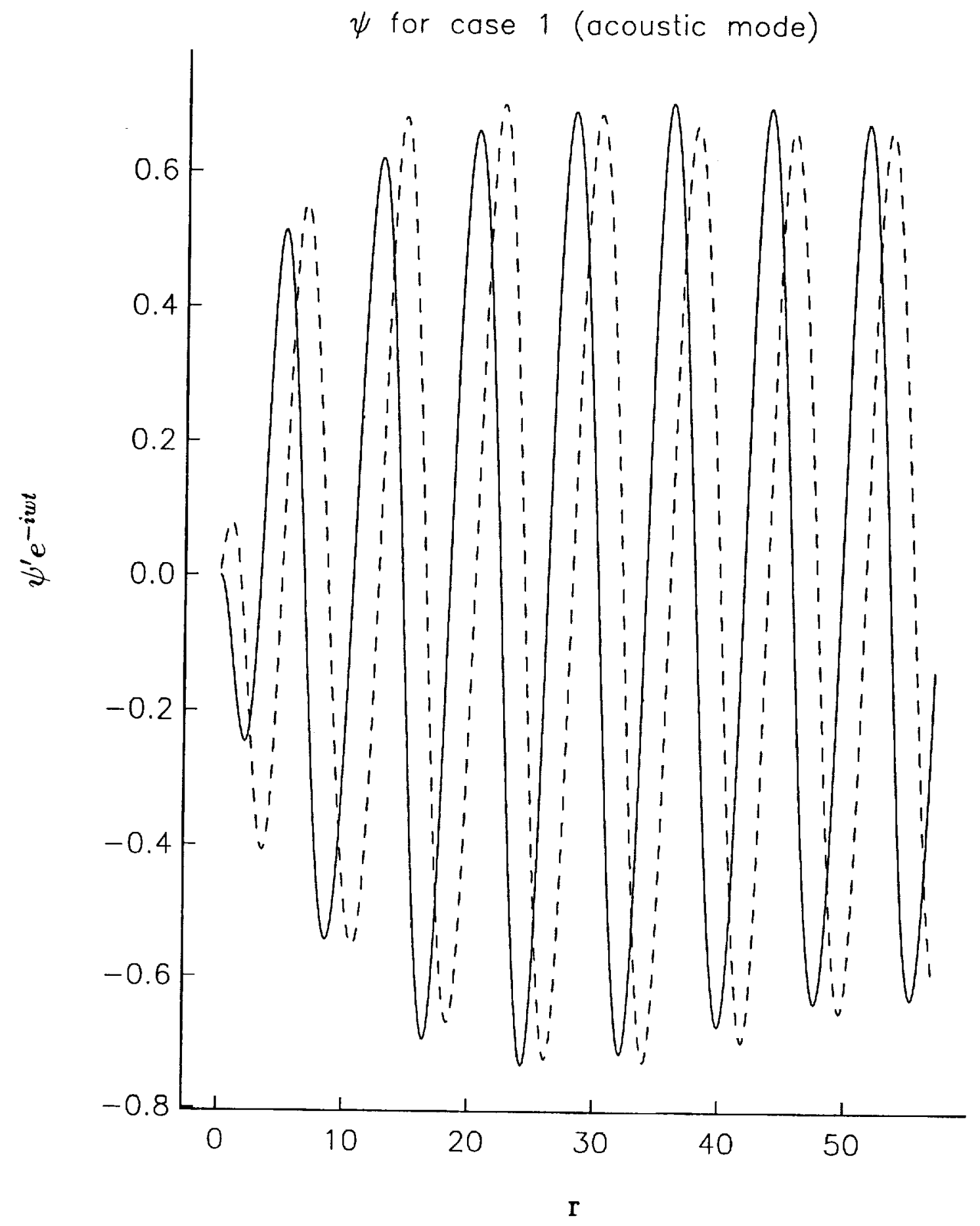

Fig.1b Shock location distribution, data as for Fig.1a. 


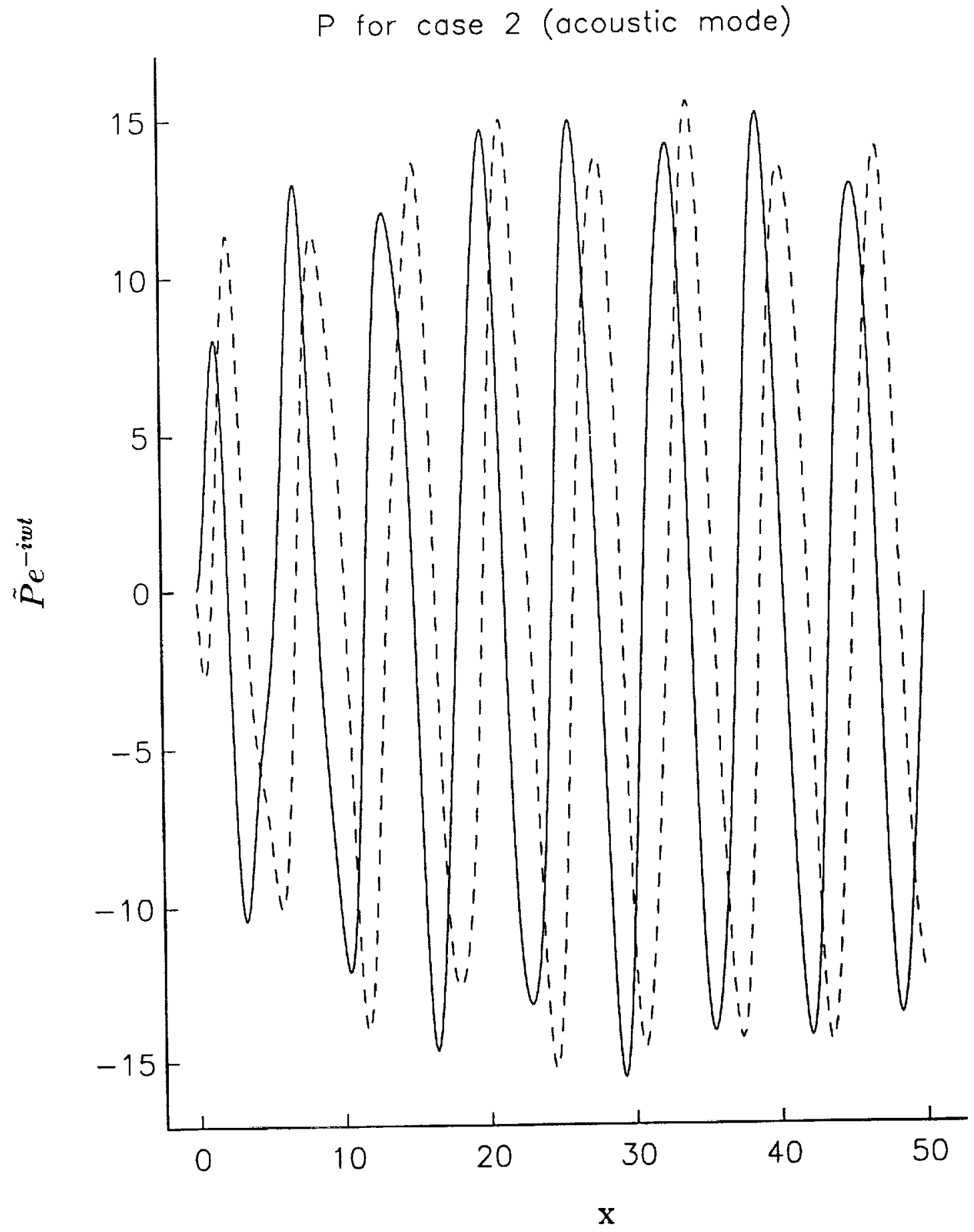

Fig.2a Wall pressure distribution for upstream fast acoustic mode, $M_{1}=5, \theta=25^{\circ}, \alpha_{1}=1$, $\alpha_{2}=0$. 


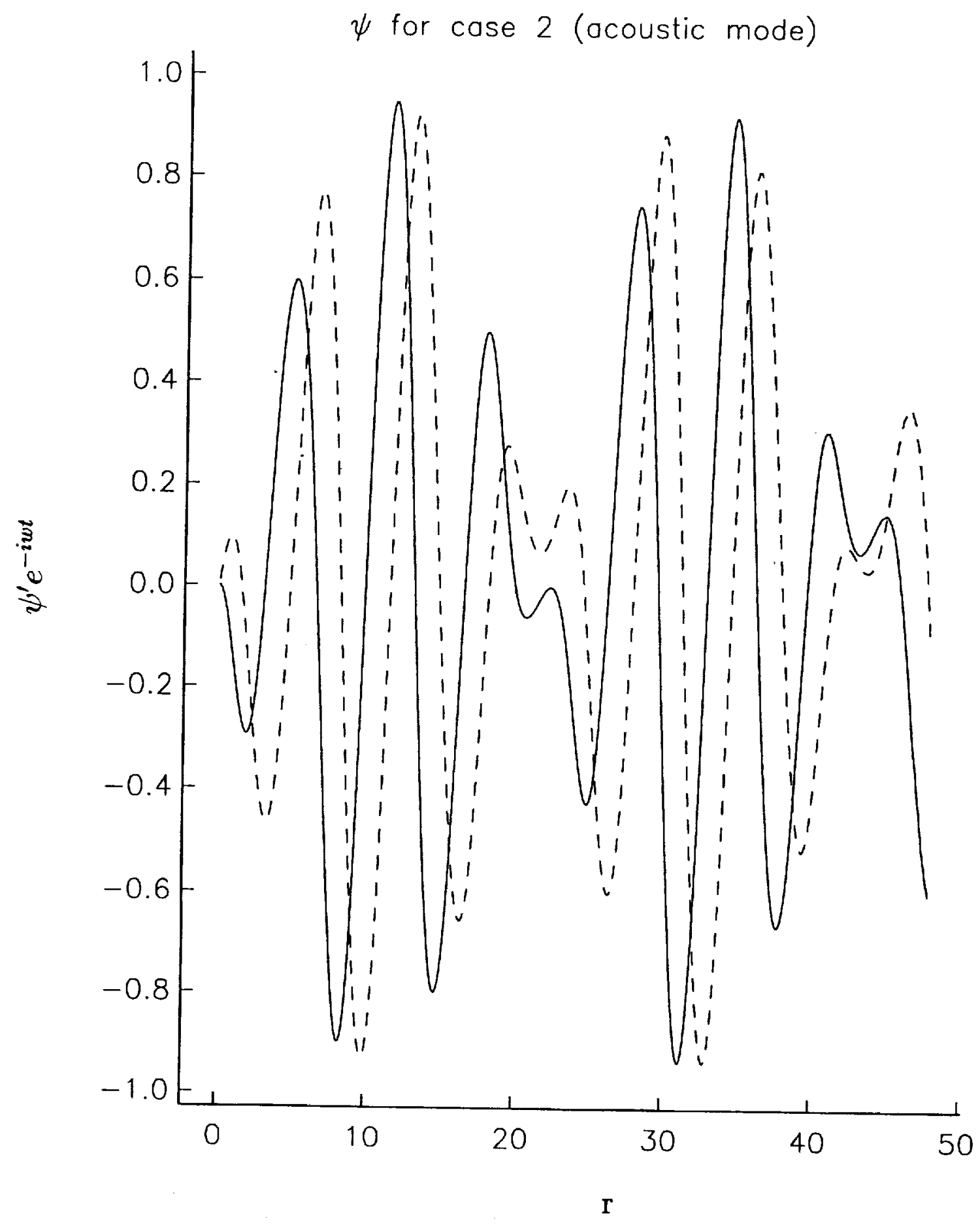

Fig.2b Shock location distribution, data as for Fig.1a. 


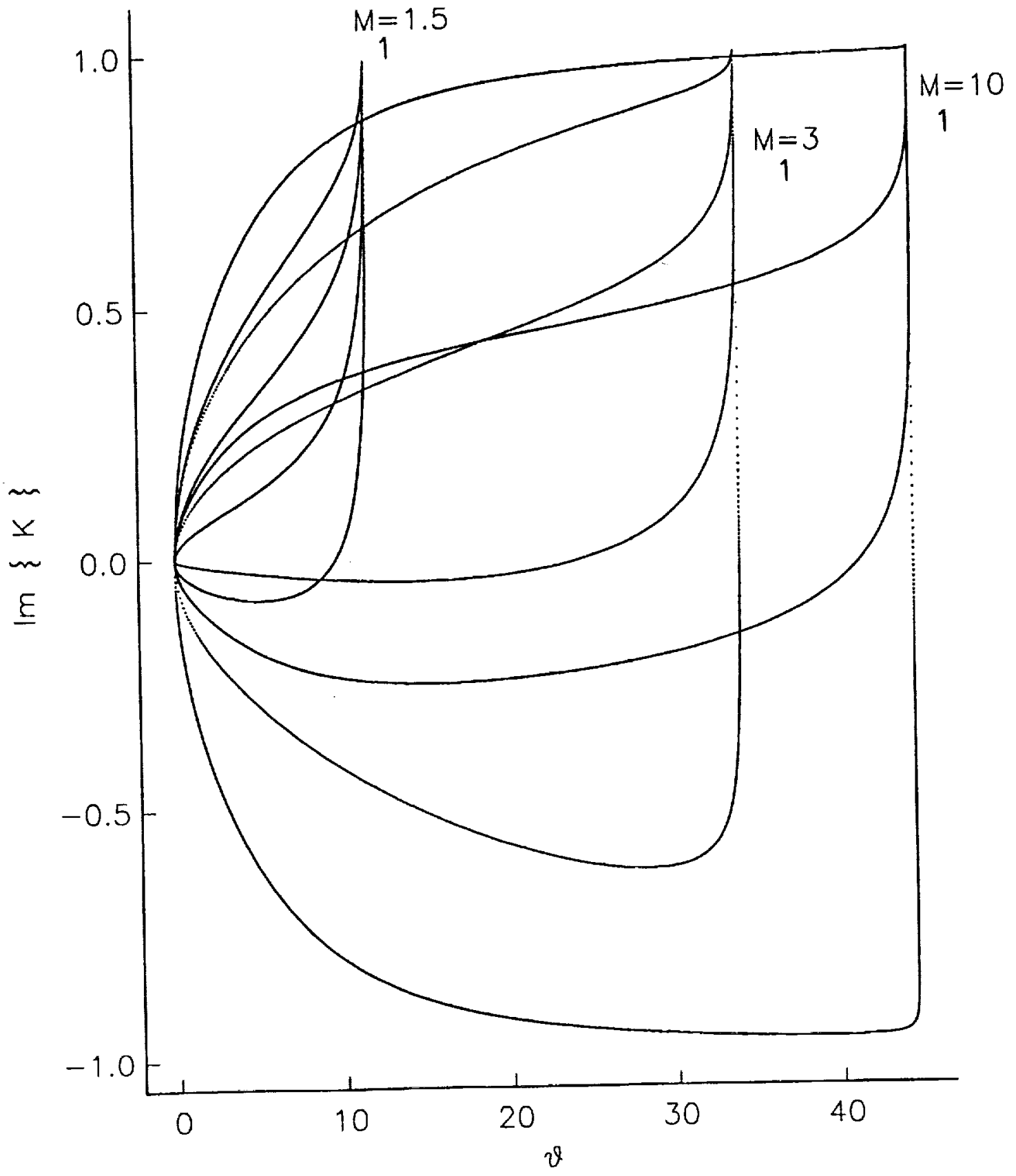

Fig.3 $\Im(K)$ for $M_{1}=1.5,3,10$. 


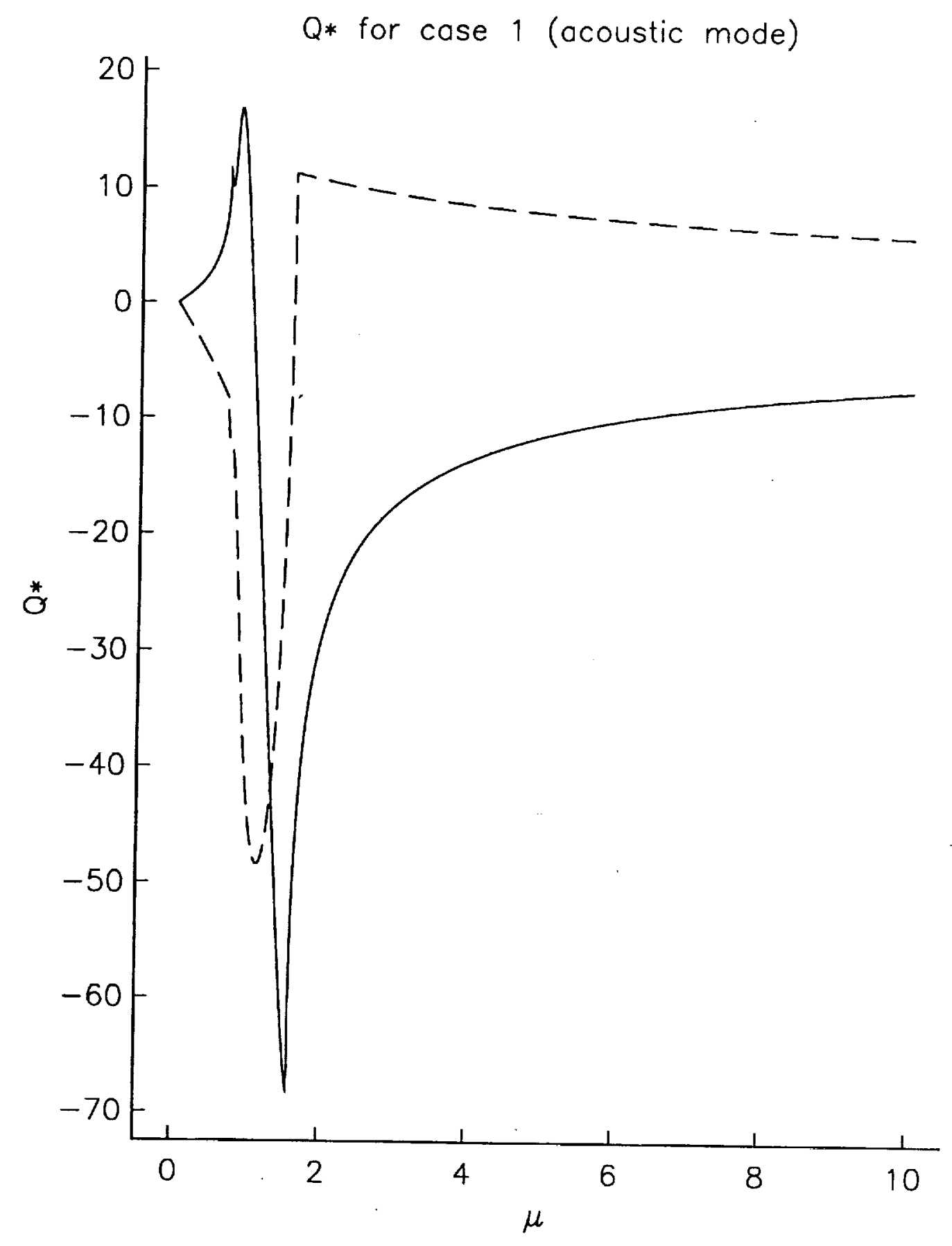

Fig.4 $Q^{*}$ for upstream slow acoustic mode, $M_{1}=5, \theta=25^{\circ}, \alpha_{1}=1, \alpha_{2}=0$. 


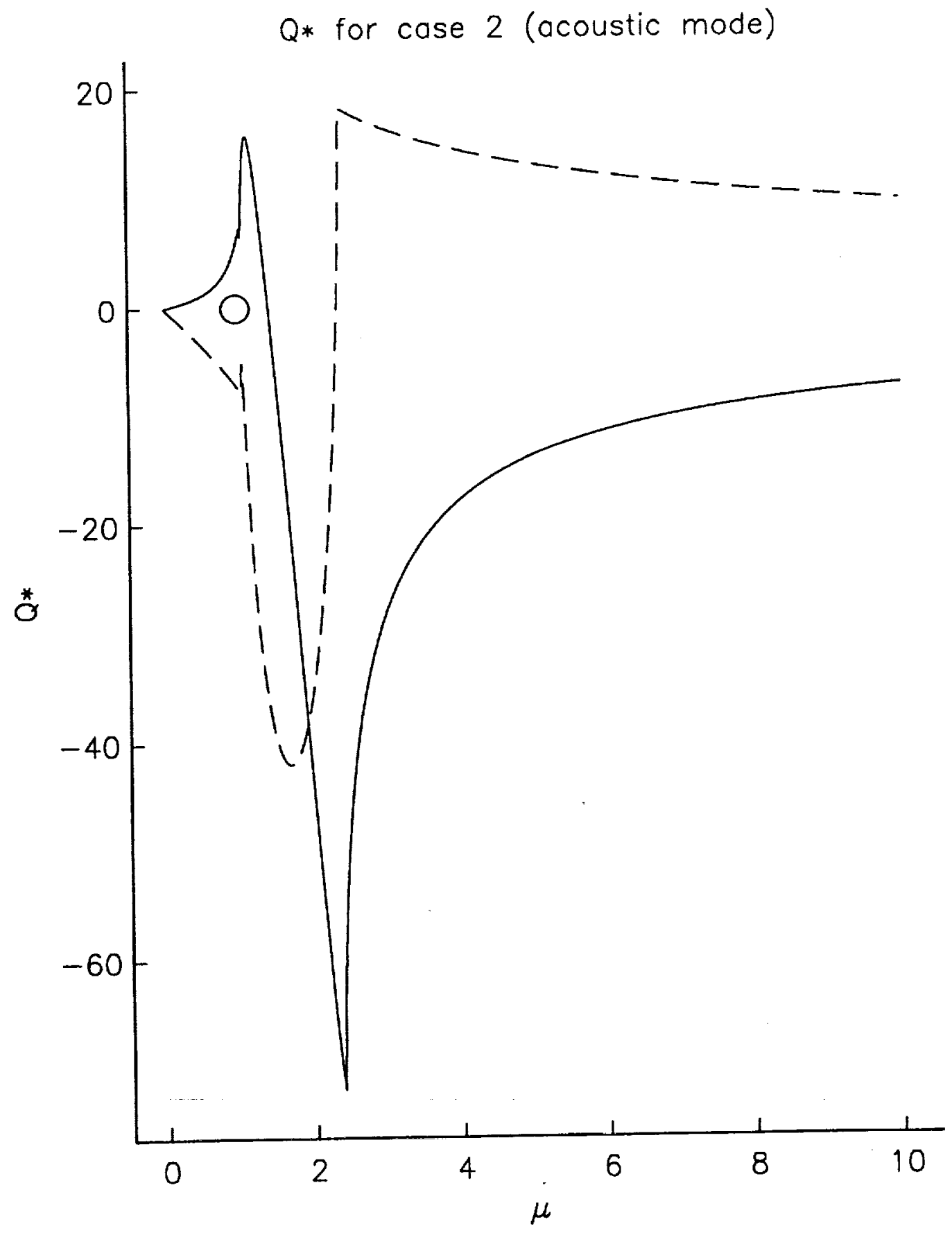

Fig.5 $Q^{*}$ for upstream fast acoustic mode, $M_{1}=5, \theta=25^{\circ}, \alpha_{1}=1, \alpha_{2}=0$. 


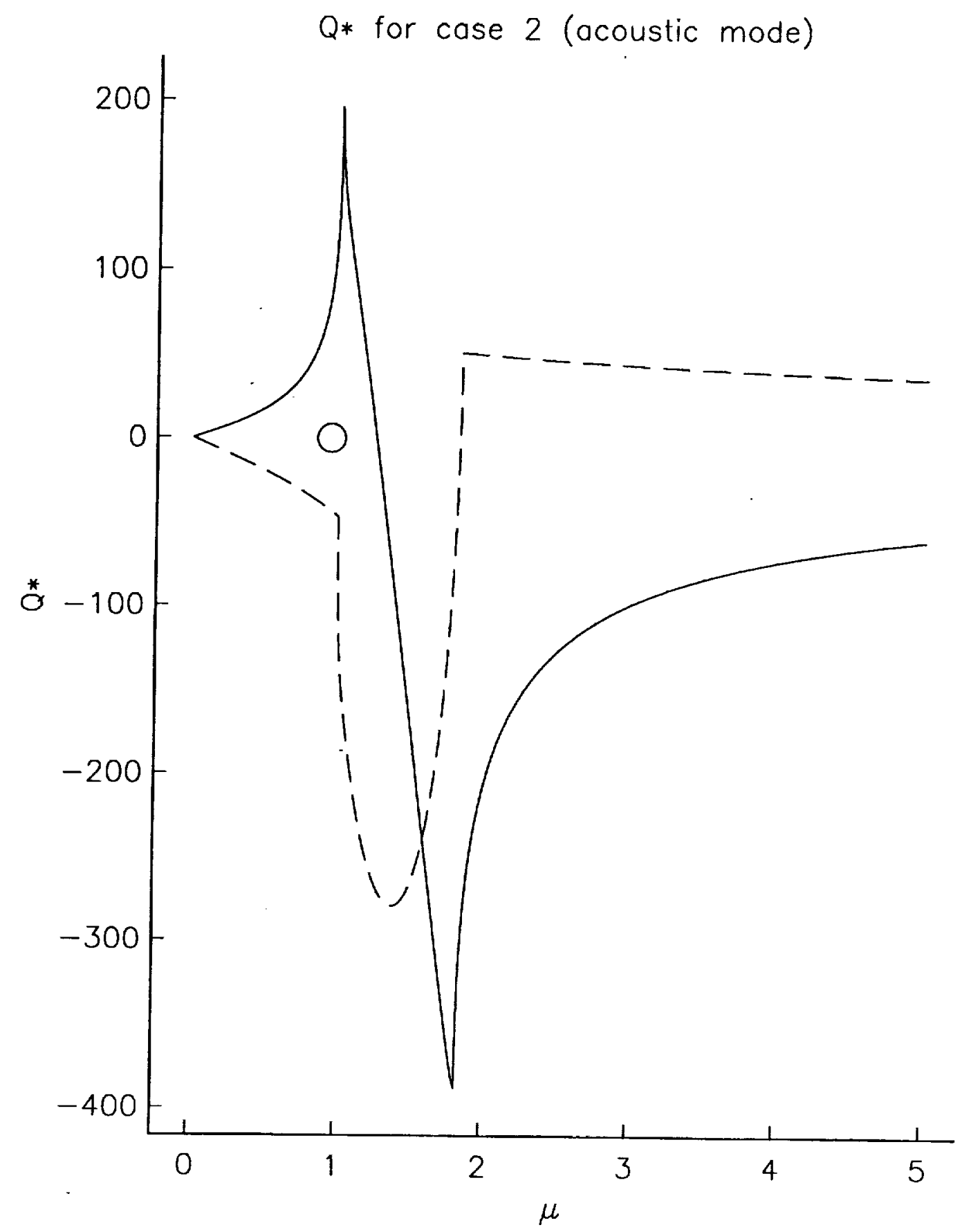

Fig.6 $Q^{*}$ for upstream fast acoustic mode, $M_{1}=10, \theta=25^{\circ}, \alpha_{1}=1, \alpha_{2}=0$. 


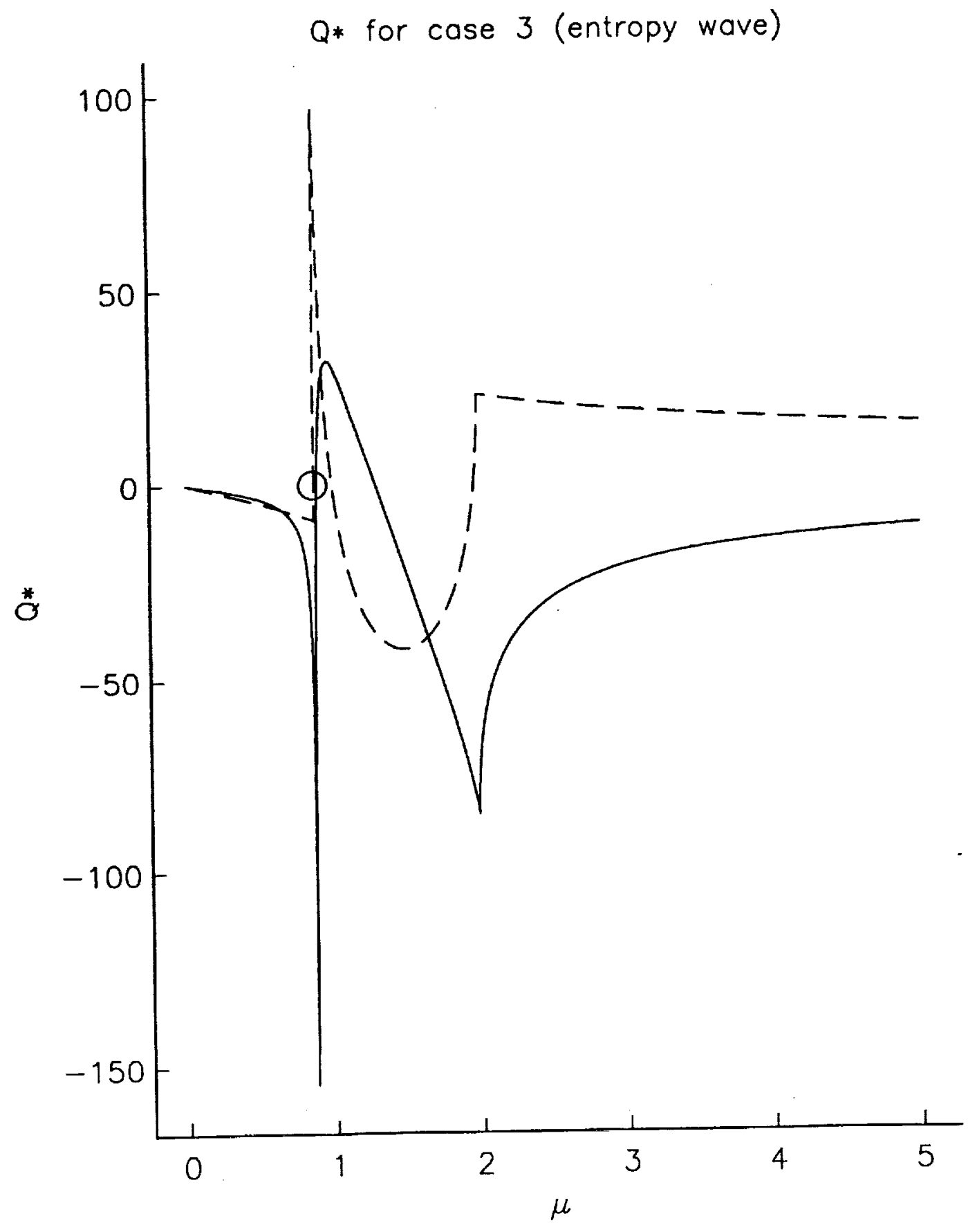

Fig.7 $Q^{*}$ for upstream entropy mode, $M_{1}=5, \theta=25^{\circ}, \alpha_{1}=1, \alpha_{2}=0$. 


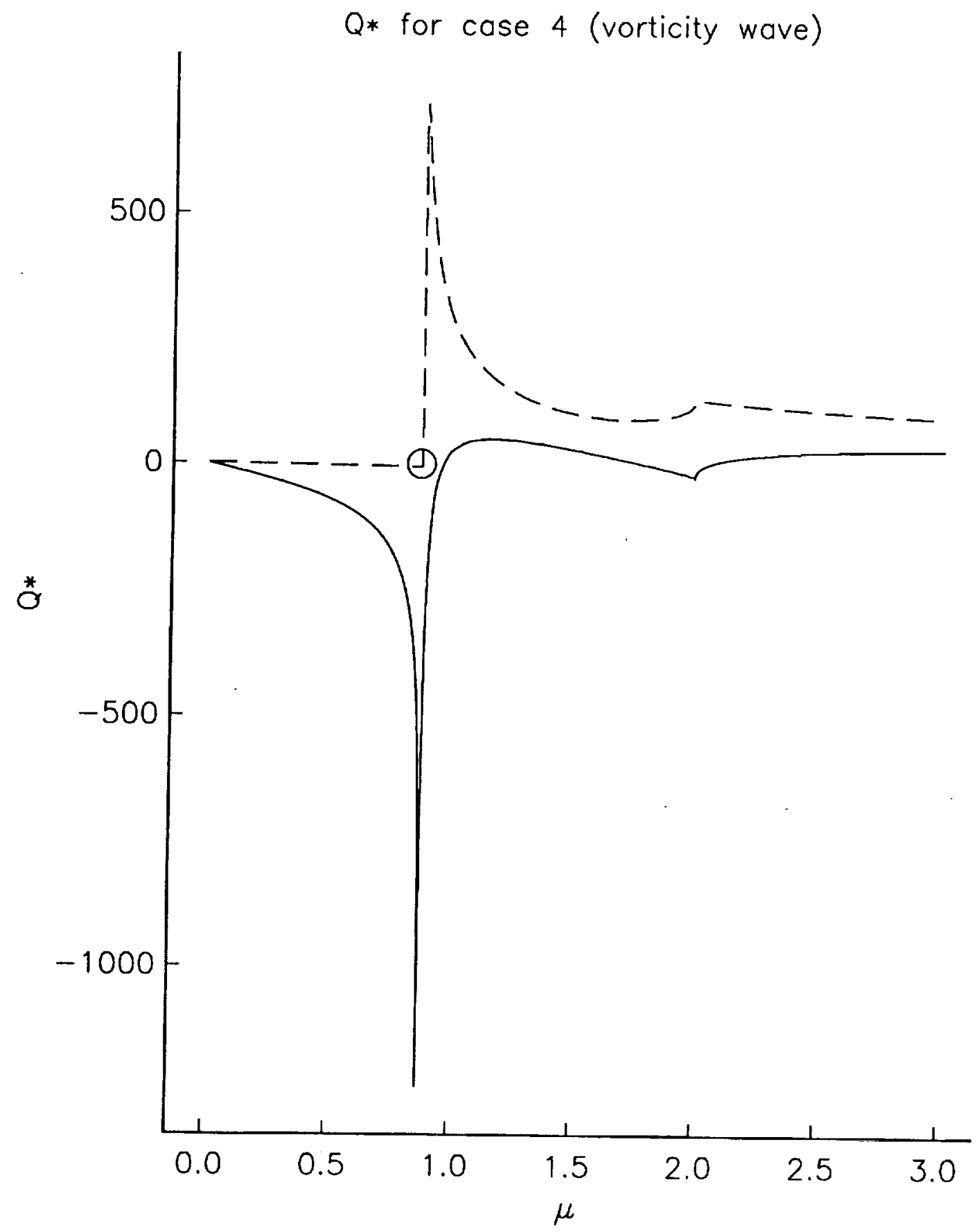

Fig.8 $Q^{*}$ for upstream vorticity mode, $M_{1}=5, \theta=25^{\circ}, \alpha_{1}=0, \alpha_{2}=1$. 


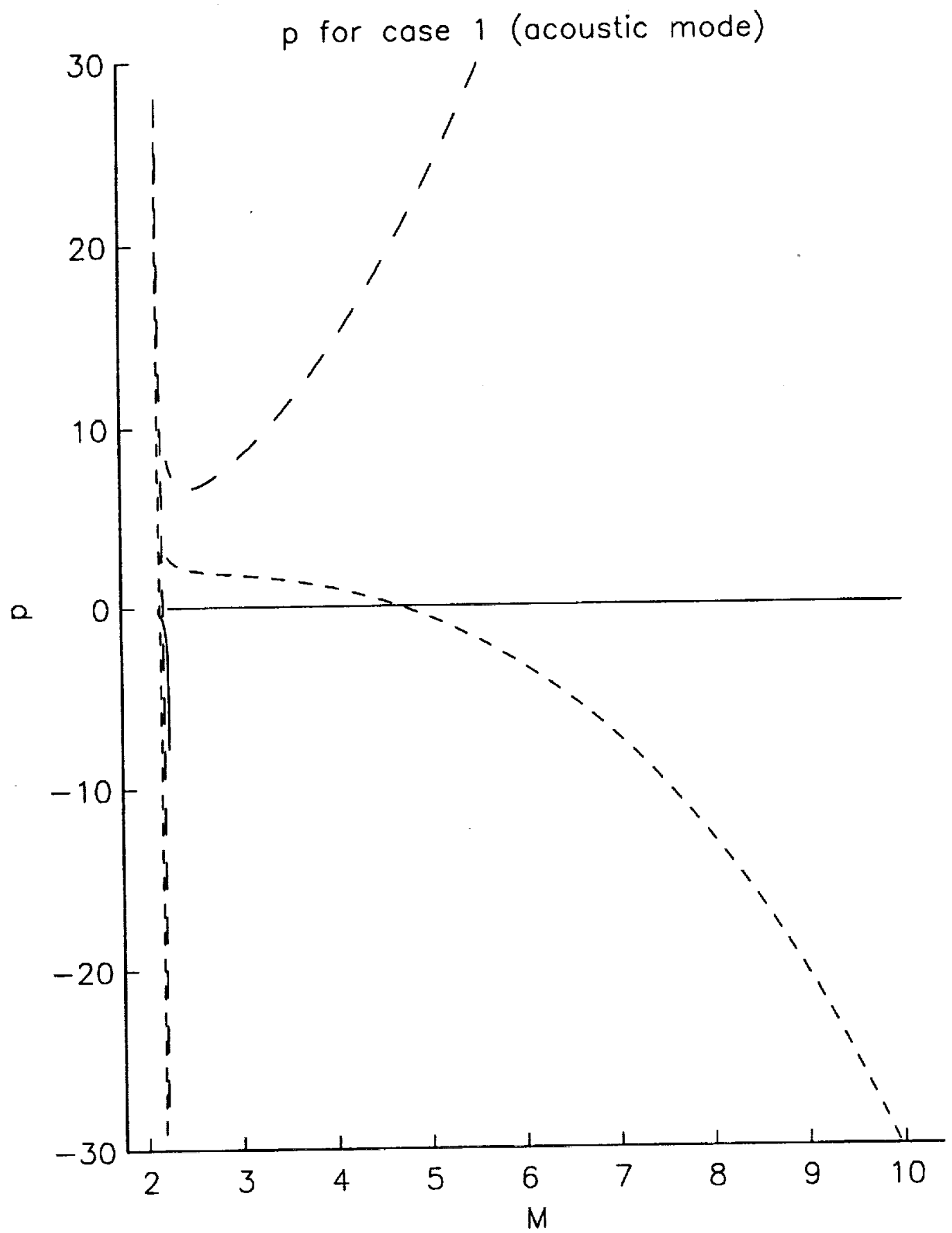

Fig.9a $P_{0}, P_{1}, P_{2}$ for upstream slow acoustic mode, $\theta=25^{\circ}, \alpha_{1}=1, \alpha_{2}=0$. 


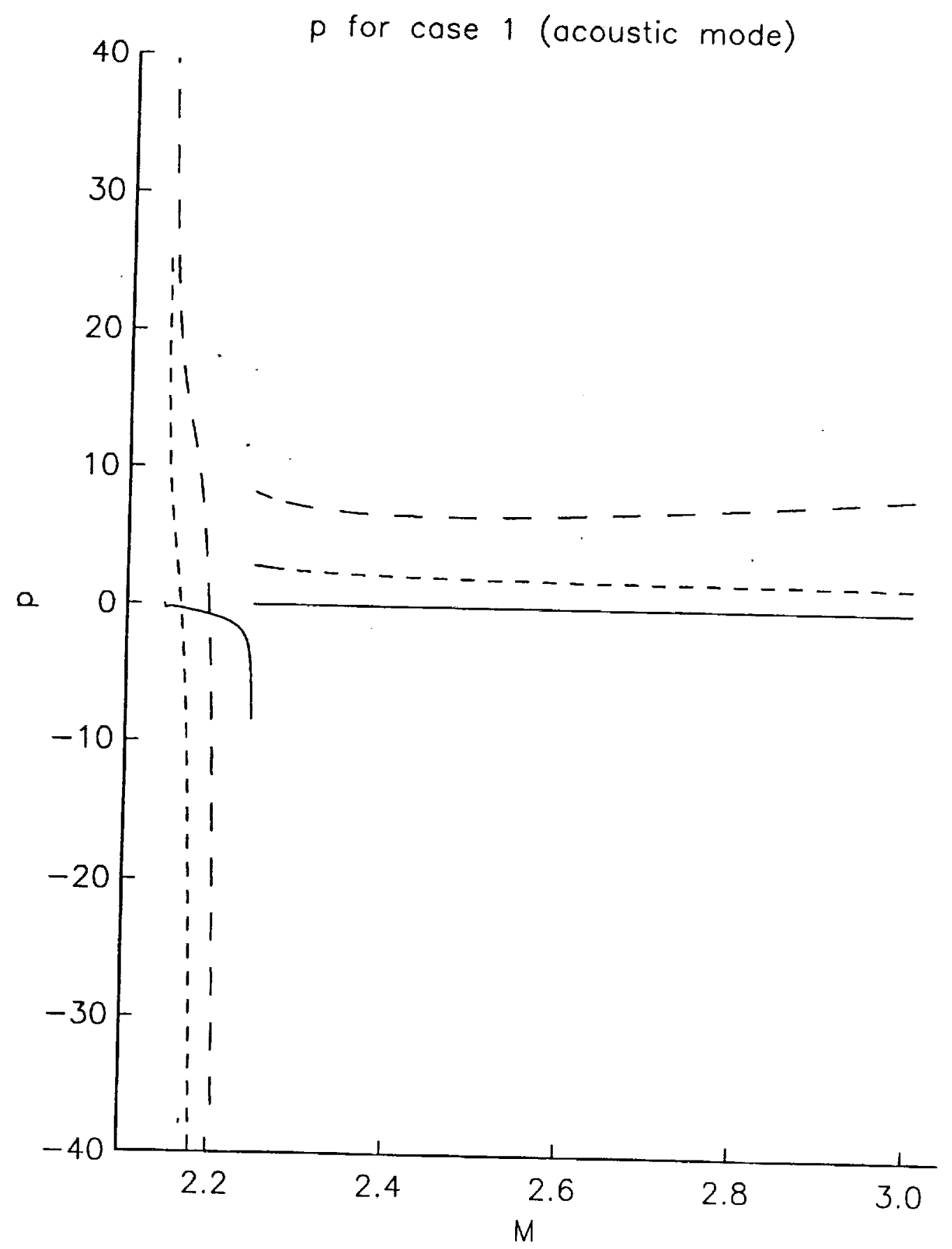

Fig.9b Details as for Fig.9a, enlarged scale. 


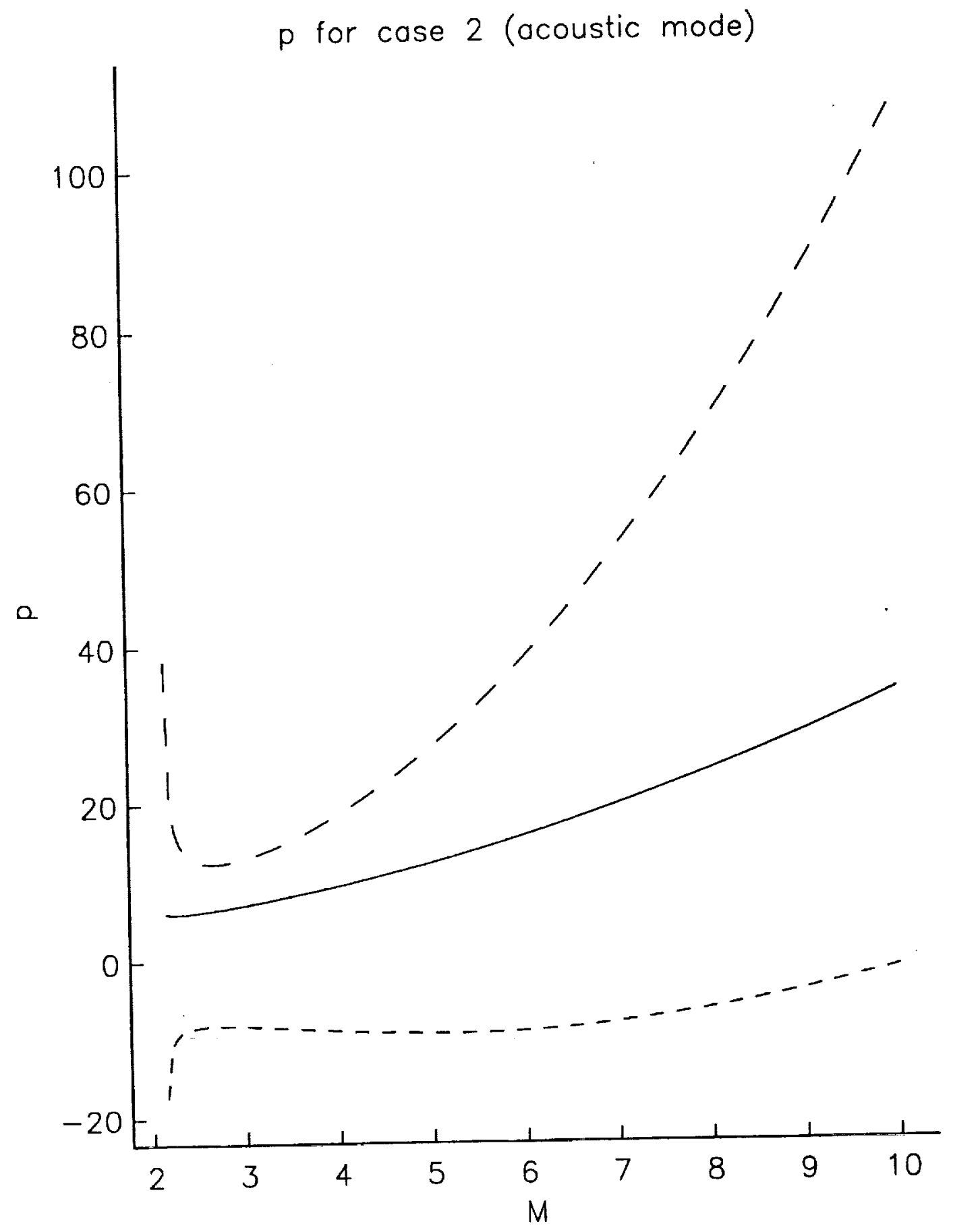

Fig.10 $P_{0}, P_{1}, P_{2}$ for upstream fast acoustic mode, $\theta=25^{\circ}, \alpha_{1}=1, \alpha_{2}=0$. 


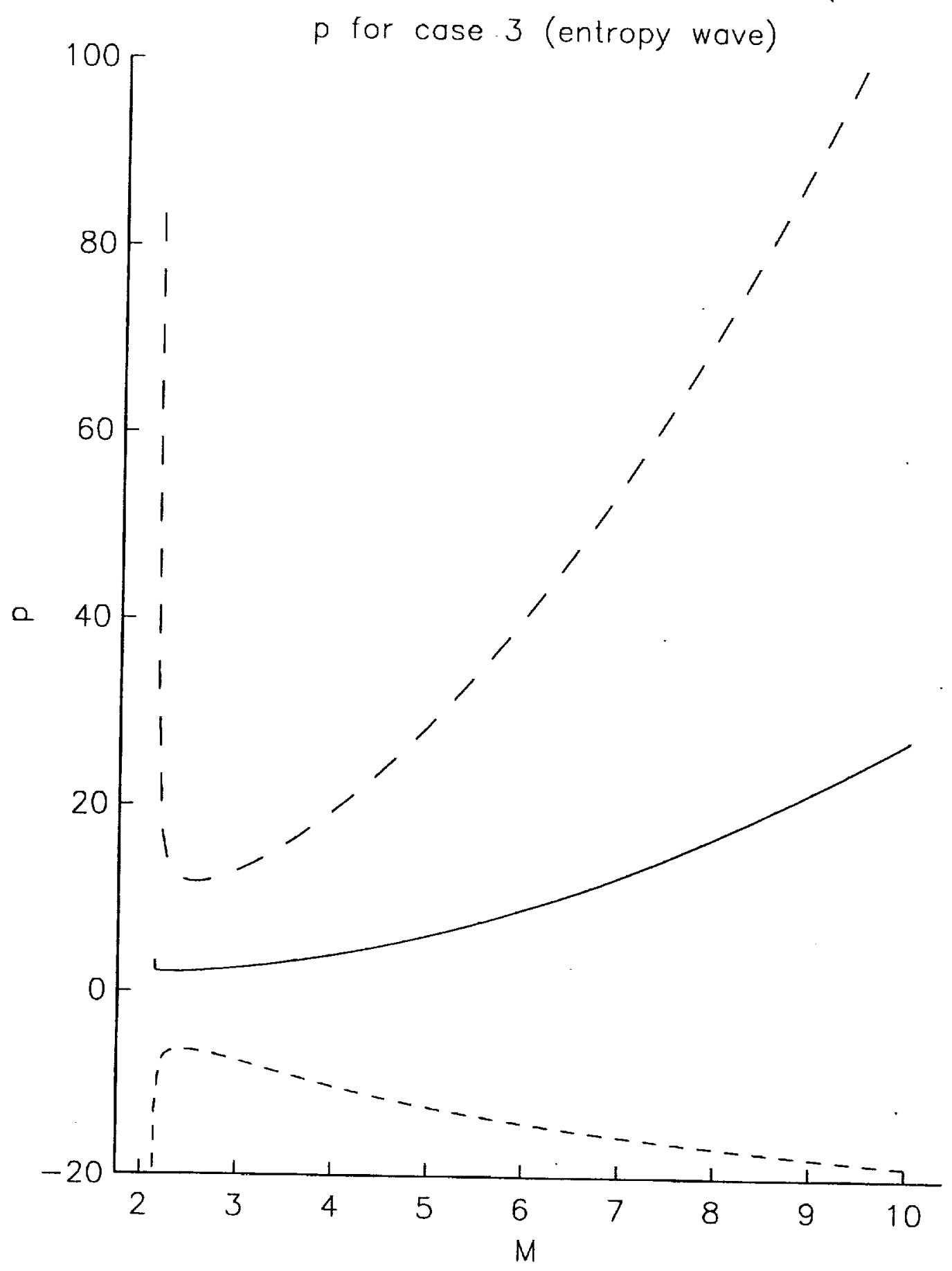

Fig.11 $P_{0}, P_{1}, P_{2}$ for upstream entropy mode, $\theta=25^{\circ}, \alpha_{1}=1, \alpha_{2}=0$. 


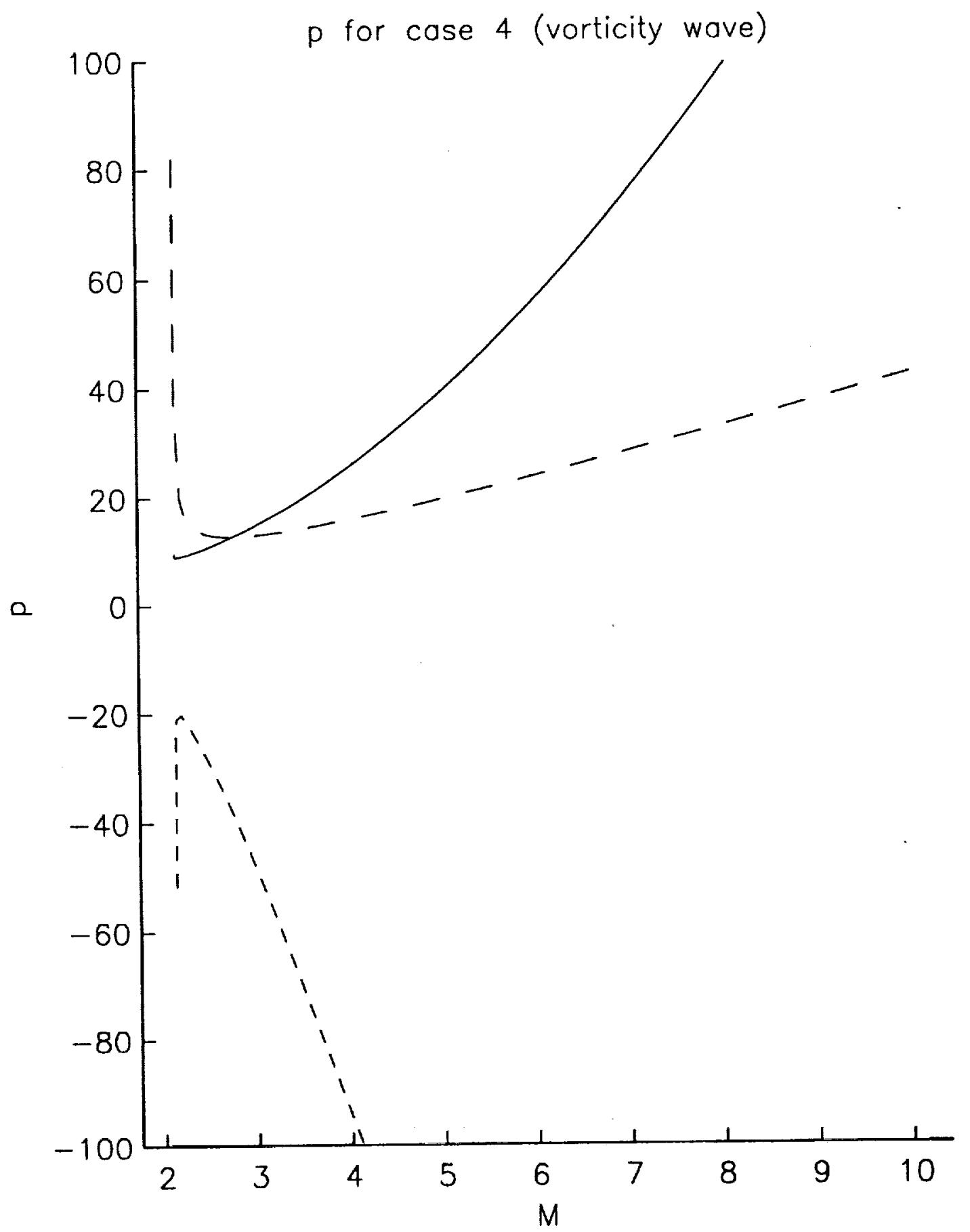

Fig.12 $P_{0}, P_{1}, P_{2}$ for upstream vorticity mode, $\theta=25^{\circ}, \alpha_{1}=1, \alpha_{2}=0$. 


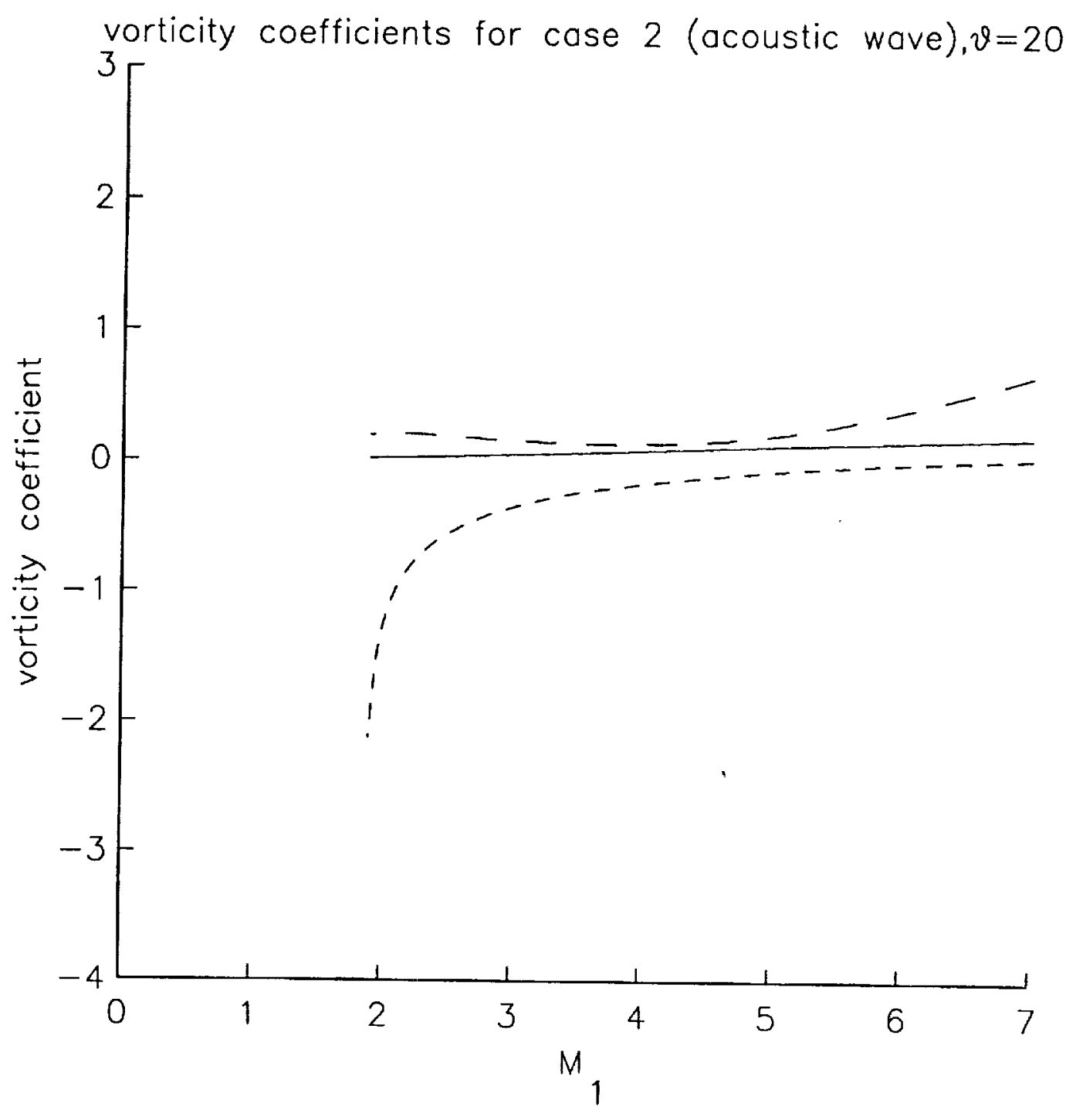

Fig.13 $\Omega_{0}, \Omega_{1}, \Omega_{2}$ for upstream fast acoustic mode, $\theta=20^{\circ}, \alpha_{1}=1, \alpha_{2}=0$. 


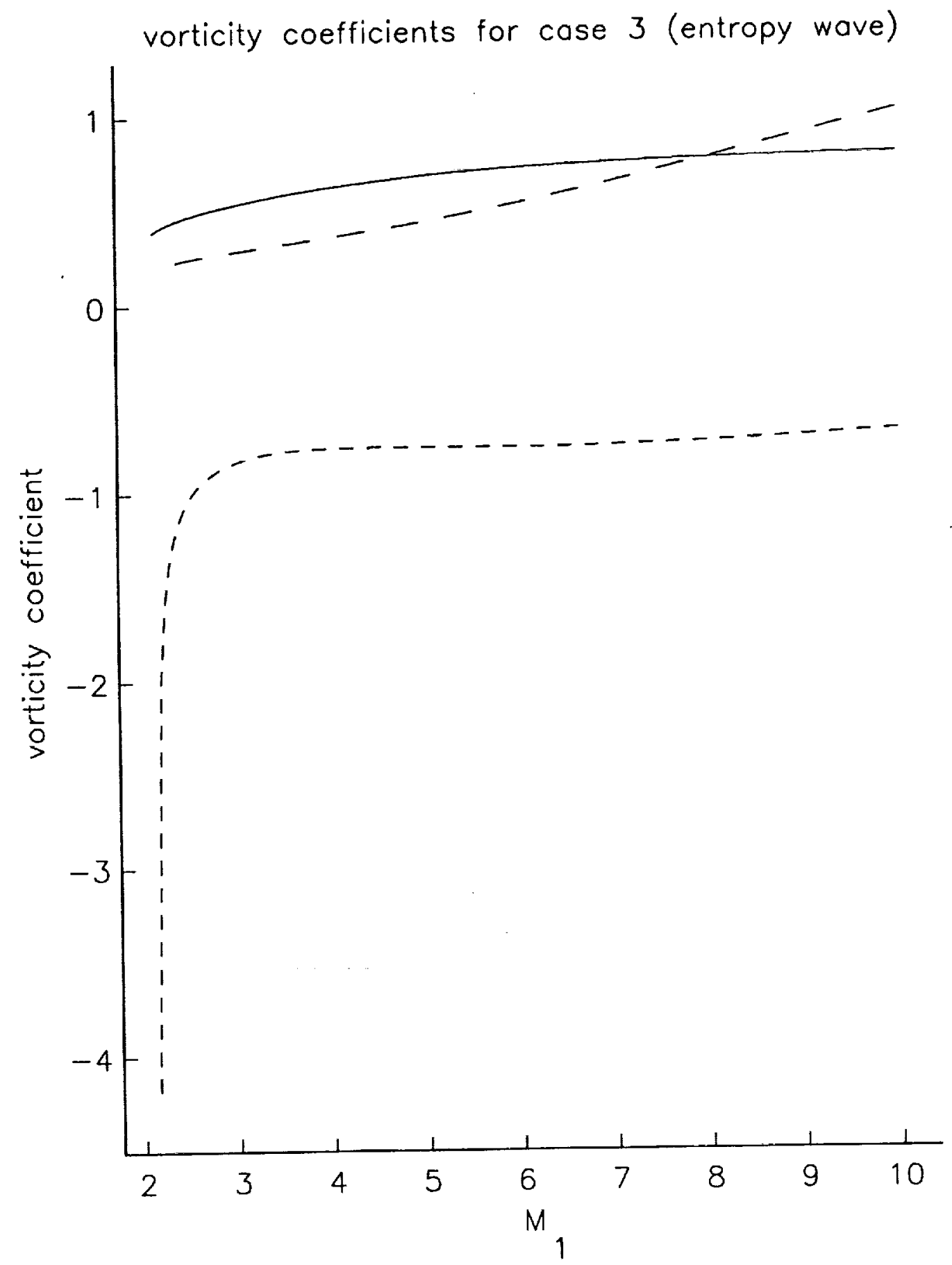

Fig.14 $\Omega_{0}, \Omega_{1}, \Omega_{2}$ for upstream entropy mode, $\theta=25^{\circ}, \alpha_{1}=1, \alpha_{2}=0$. 


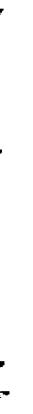




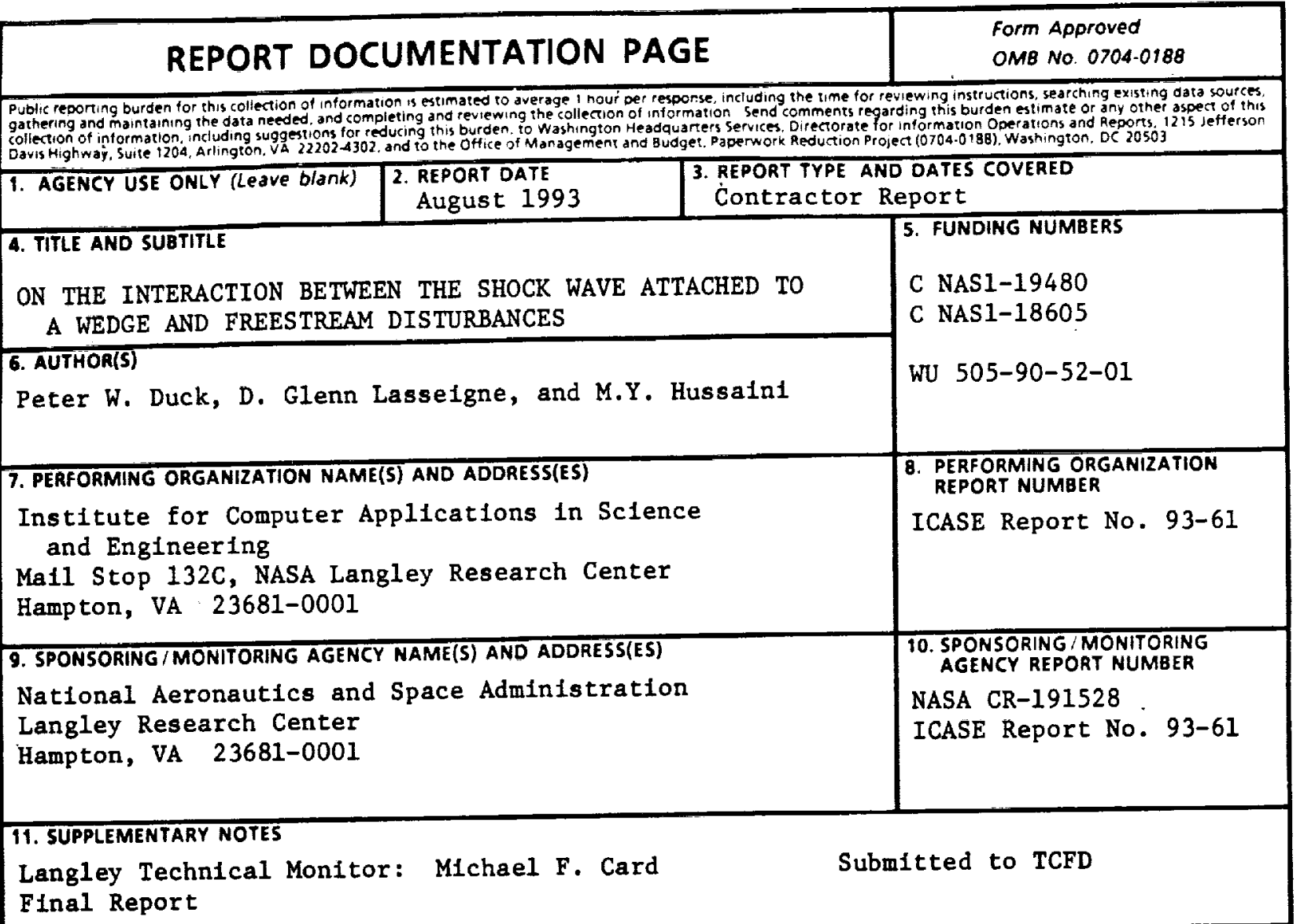

12a. OISTRIBUTION/AVALABILITY STATEMENT
Unclassified - Unlimited
Subject Category 34

\begin{tabular}{|c|c|c|c|}
\hline \multicolumn{3}{|c|}{$\begin{array}{l}\text { 14. SUBJECT TERMS } \\
\text { shock wave, free stream disturbances }\end{array}$} & \multirow{2}{*}{$\begin{array}{l}\text { 15. NUMBER OF PAGES } \\
48 \\
\begin{array}{l}\text { 16. PRICE COOE } \\
\text { AO3 }\end{array} \\
\text { 20. LIMITATION OF ABSTRACT }\end{array}$} \\
\hline $\begin{array}{l}\text { 17. SECURITY CLASSIFICATION } \\
\text { OF REPORT } \\
\text { Unclass if } 1 \text { ed }\end{array}$ & $\begin{array}{l}\text { 18. SECURITY CLASSIFICATION } \\
\text { OF THIS PAGE } \\
\text { Unclass } 1 \text { fied }\end{array}$ & $\begin{array}{l}\text { 19. SECURITY CLASSIFICATION } \\
\text { OF ABSTRACT }\end{array}$ & \\
\hline
\end{tabular}


National Aeronautics and

Space Administration

Code JTT

Washington, D.C.

20546-0001

BULK RATE

POSTAGE \& FEES PAID

NASA

Official Busıness

Permit No. G-27

Penalty for Private Use, $\$ 300$ 